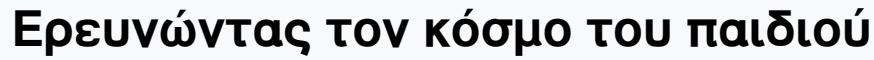

Tóp. 8 (2008)

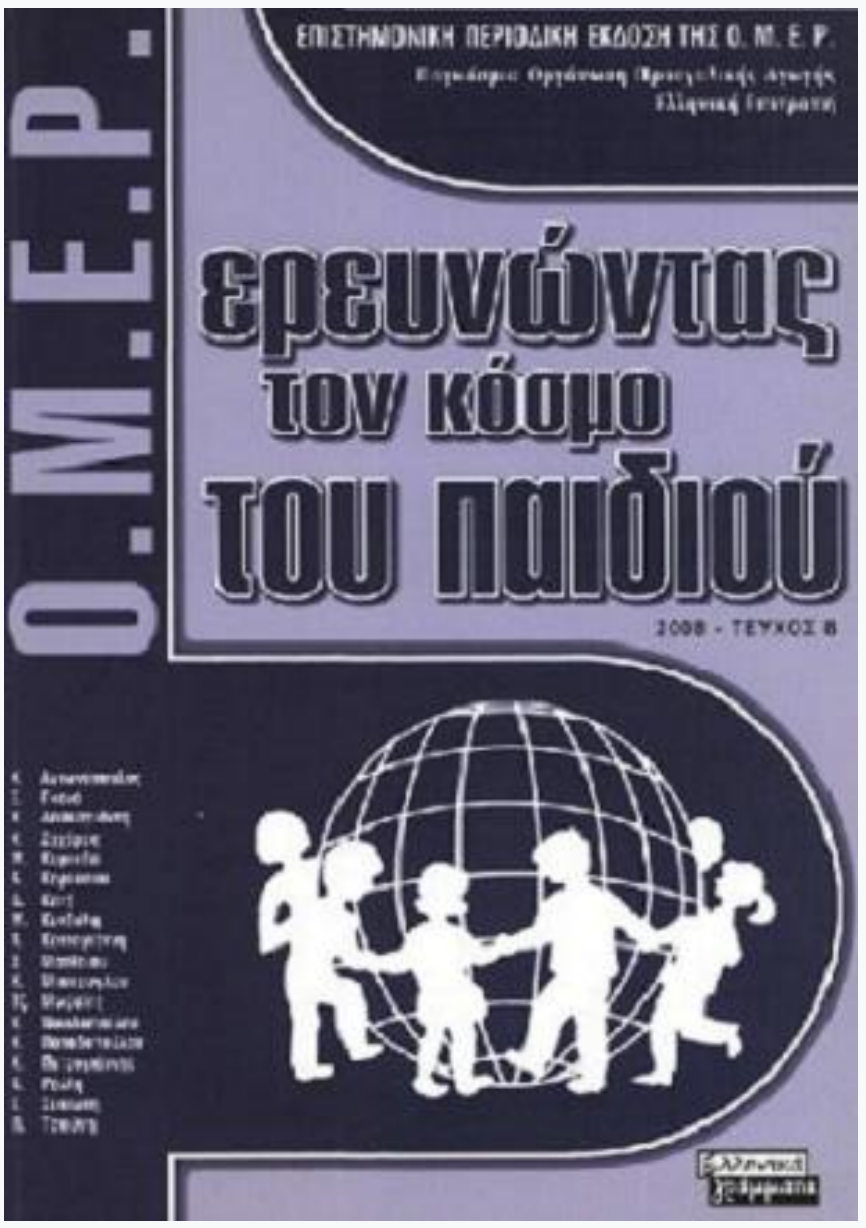

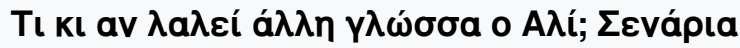

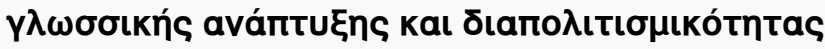

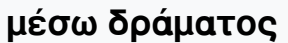

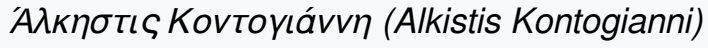

doi: $\underline{10.12681 / \mathrm{icw} .18205}$

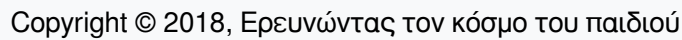

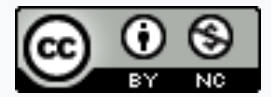

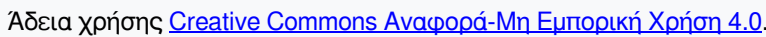

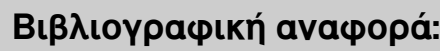

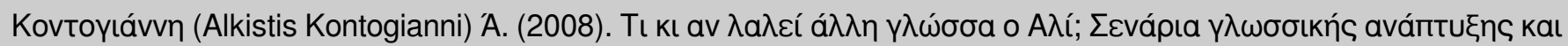

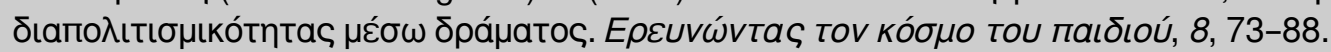

https://doi.org/10.12681/icw.18205 


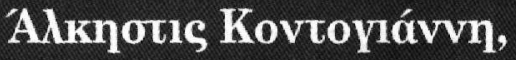

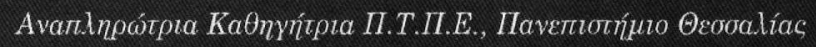

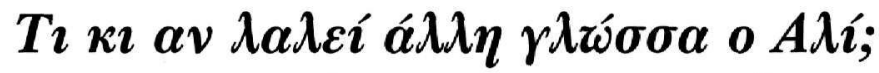

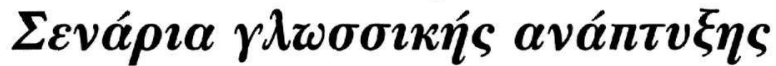

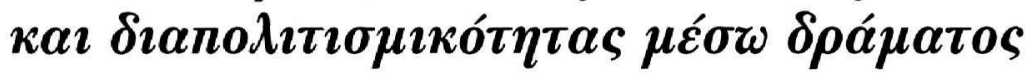

\section{Пері $\mathbf{n} \Psi \mathbf{n}$}

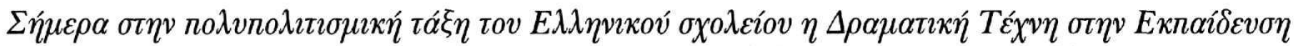

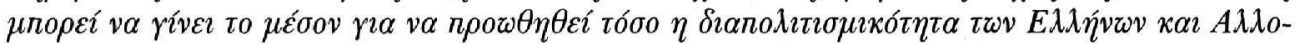

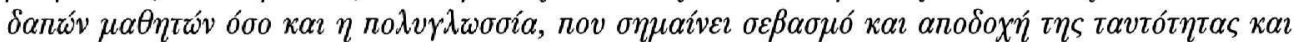

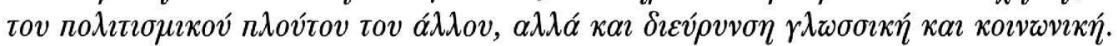

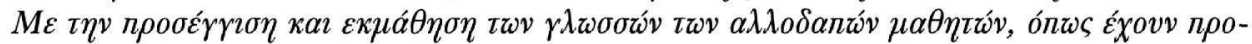

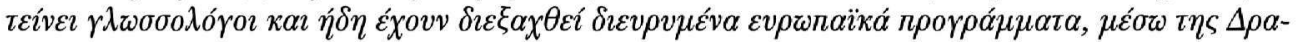

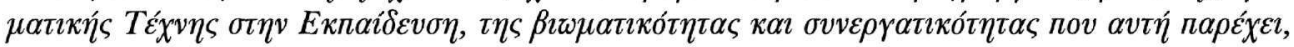

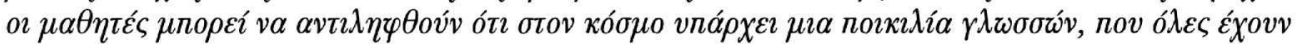

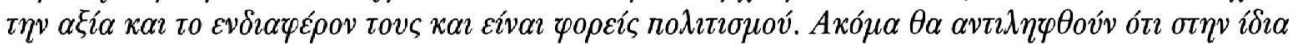

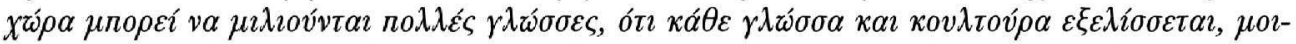

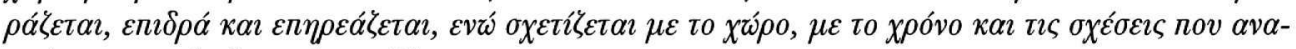

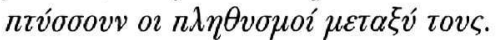

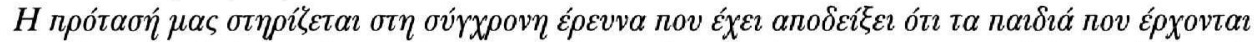

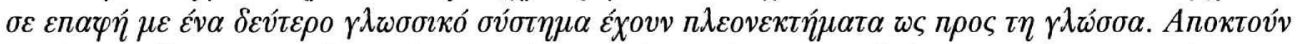

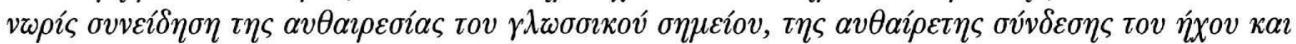

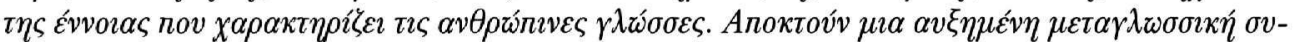

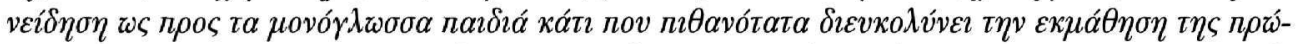

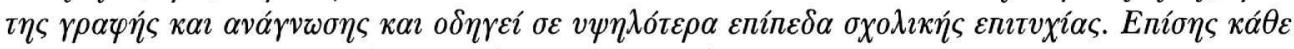

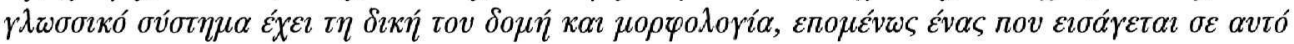

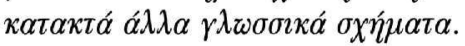

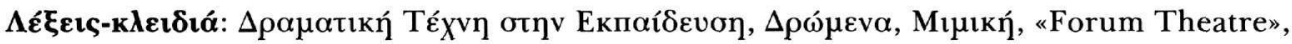

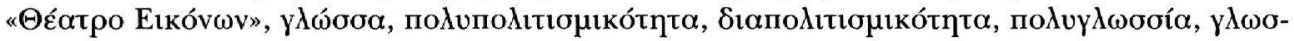

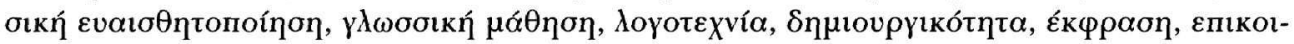

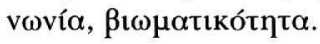




\section{Plots for language development and interculturalism through drama}

\section{Abstract}

Drama in Education in today's multicultural Greek School classrooms can be the means to promote an inter-cultural attitude among Greek and foreign students, as well as multilingualism, hence also an acceptance and respect of one another's identity and cultural wealth along with a widening of one's social and language skills.

It has been a suggestion of linguists, already taken up within broadened European programs, that through Drama in Education and the collaborative, experience-based learning it offers, students may realize that there exists a variety of languages in the world, each valuable and interesting in its own right, and a carrier of civilization. Students will also realize that many different languages can be spoken within the same country; that each language and culture evolve, share, influence and become influenced, while in close association to the place, the times and the relations that develop among populations.

Our proposal rests on an established conclusion of modern research, namely that children that have been in contact with a second linguistic framework stand in comparative advantage as to their language skills. They become aware at an early stage of the arbitrariness of linguistic signs and symbols, of the arbitrary connection between the sound and the meaning which characterizes human languages. They attain a meta-linguistic awareness higher than monolingual children, which most probably assists them in learning how to read and write and leads to increased levels of school success. It also ought to be mentioned that upon being introduced to another linguistic framework, one manages to master novel linguistic constructs, as each such framework possesses its own distinct structure and morphology.

Key words: Drama in Education, Acting out, Mime, Forum Theatre, Image Theatre, Language. Multiculturalism. Interculturalism, multilingualism, language awareness, language learning, literature, creativity, expression, communication.

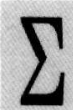

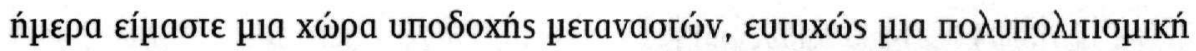

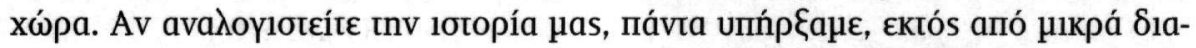

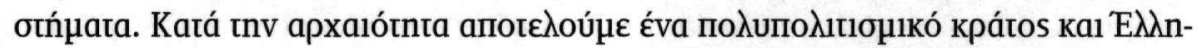

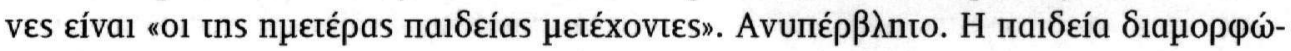

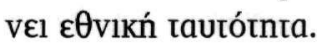

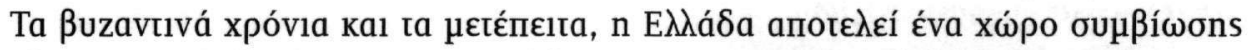

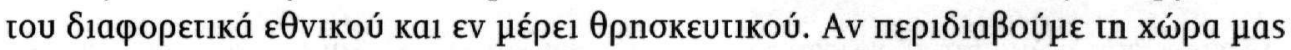

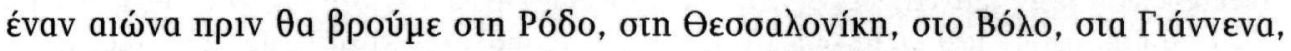

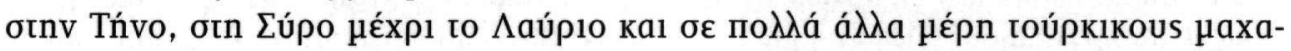

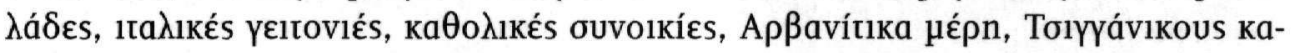

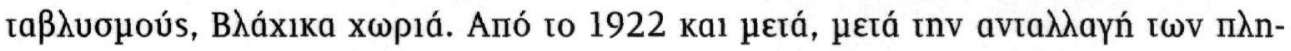

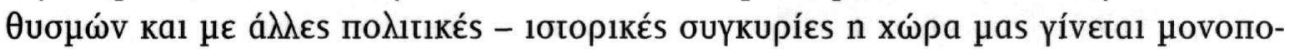

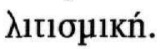

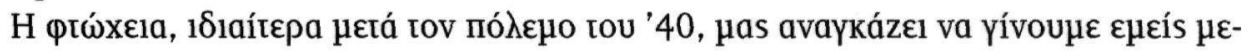




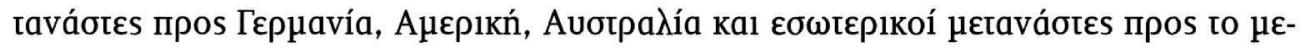
yádo áotu.

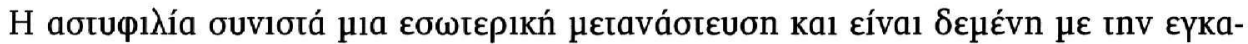

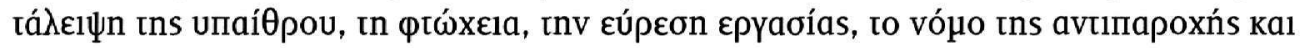

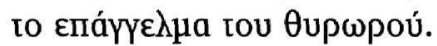

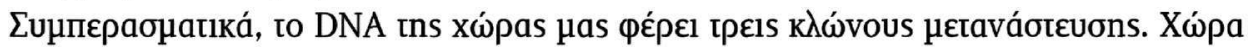

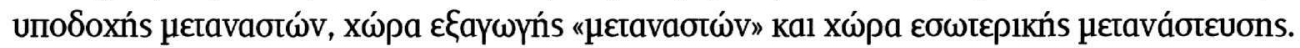

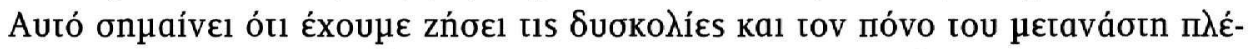

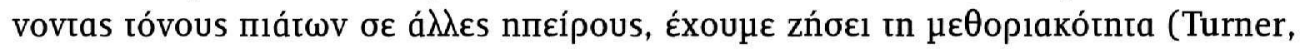

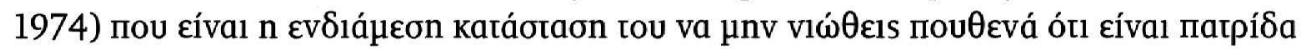

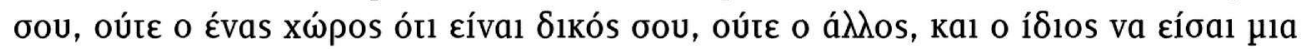

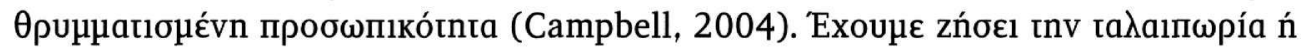

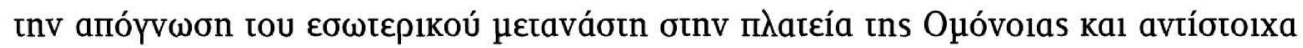

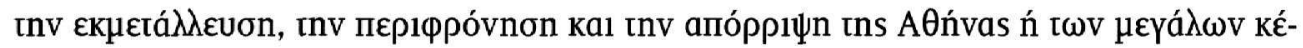

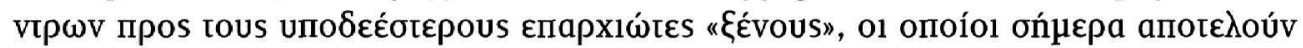

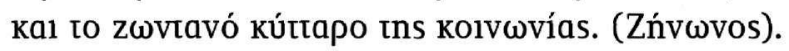

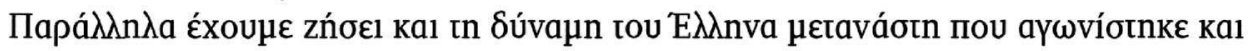

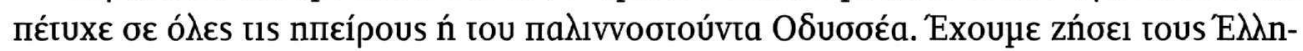

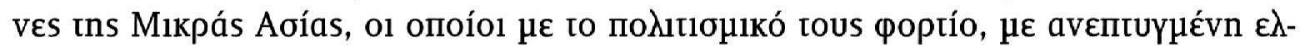

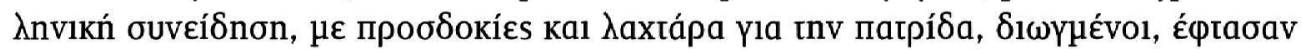

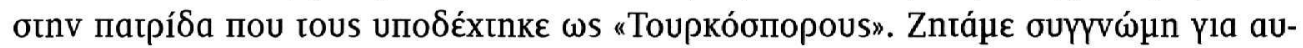

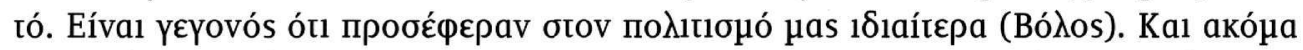

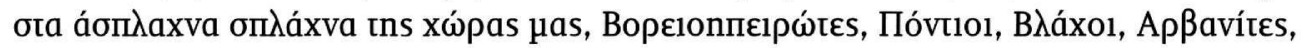

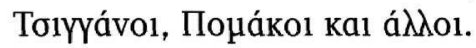

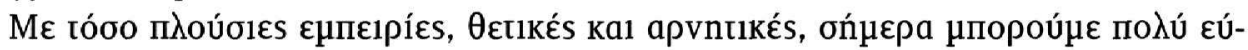

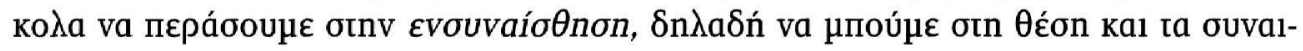

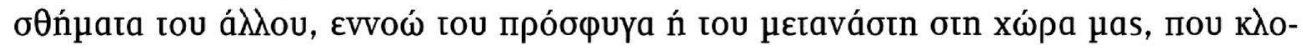

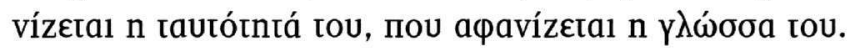

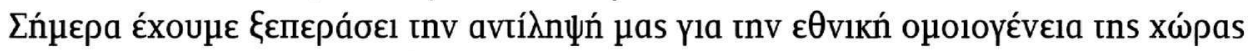

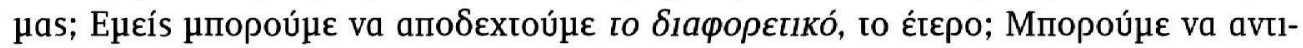

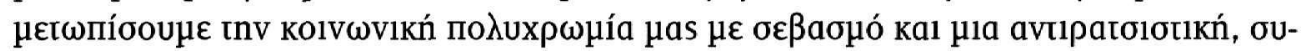

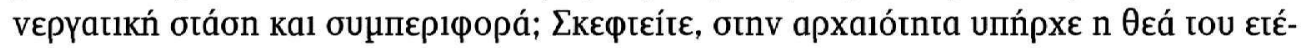

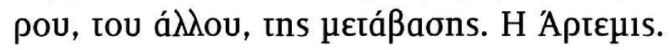

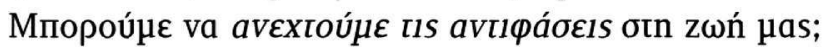

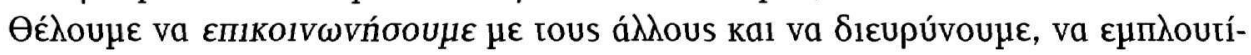

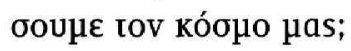

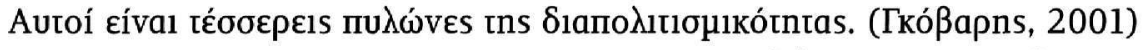

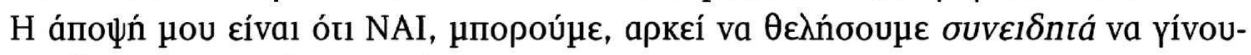

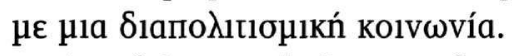

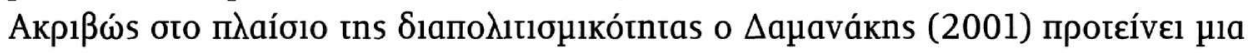

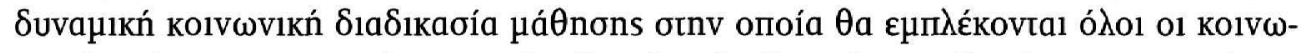

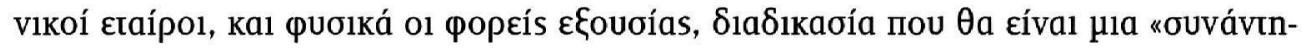

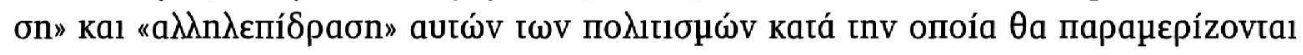




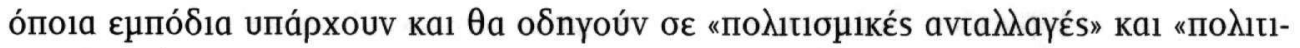

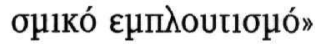

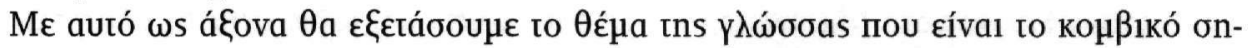

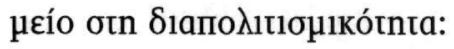

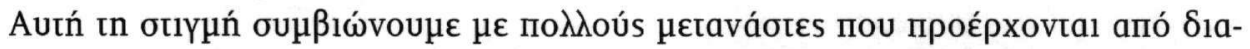

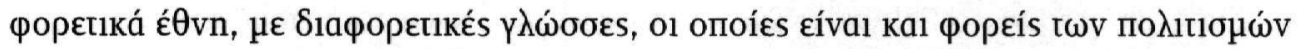

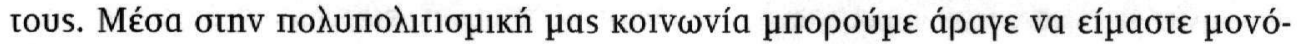

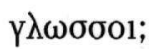

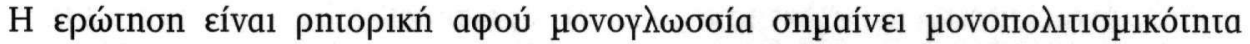

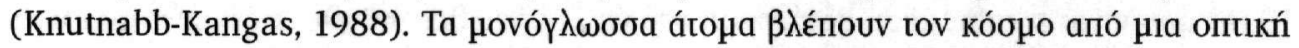

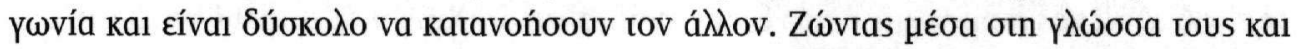

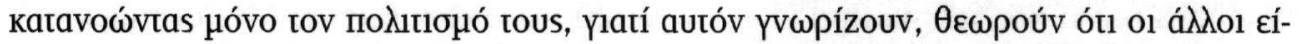

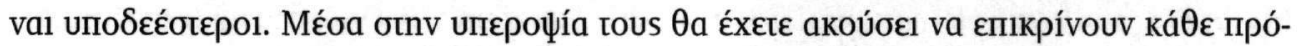

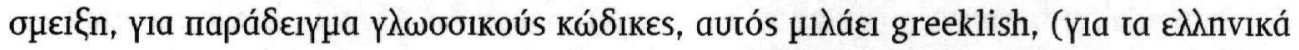

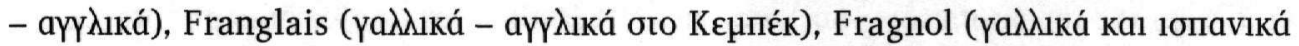

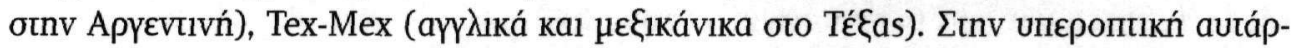

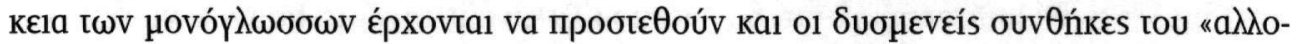

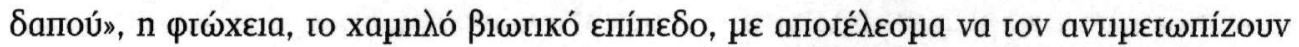

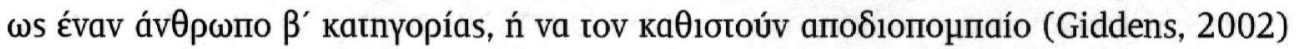

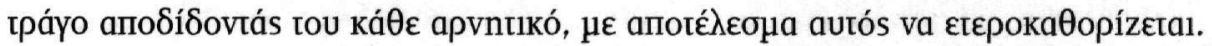

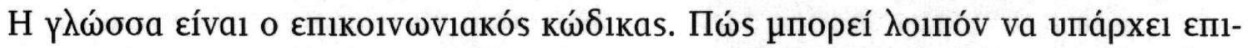

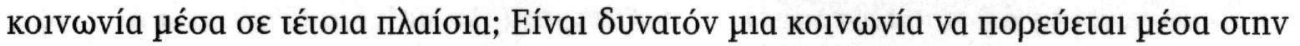

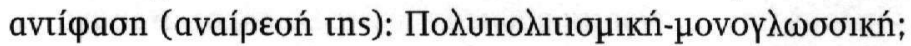

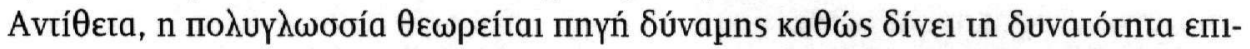

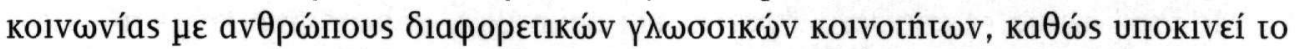

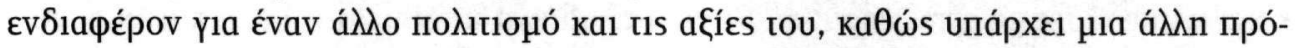

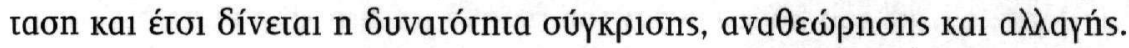

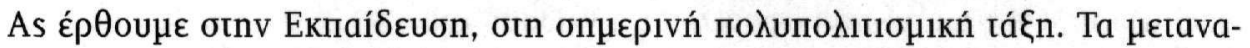

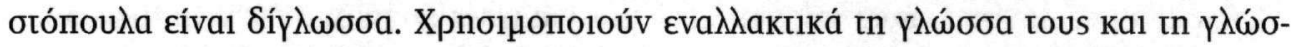

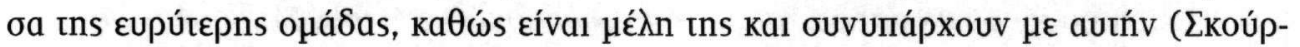

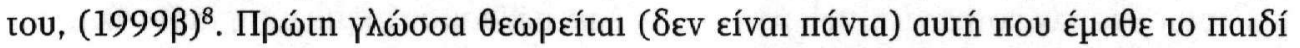

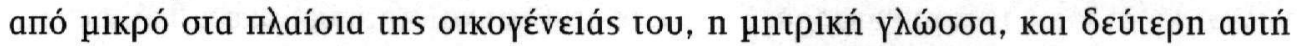

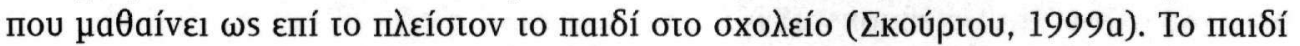

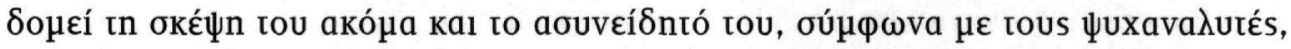

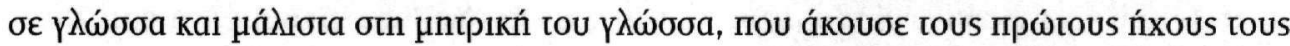

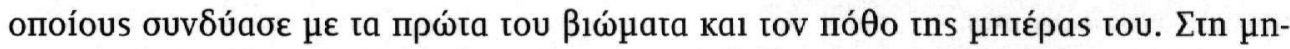

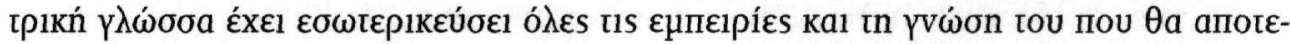

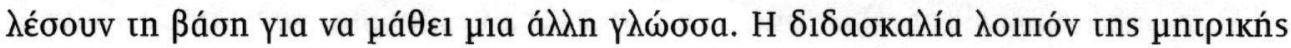

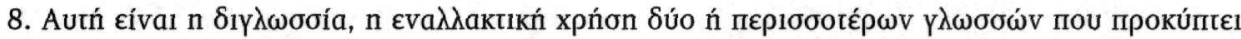

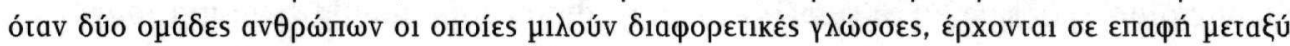

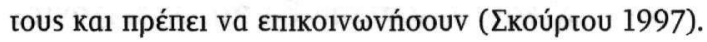




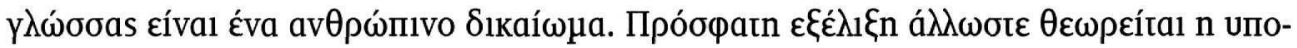

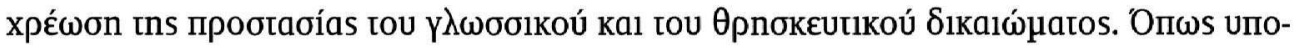

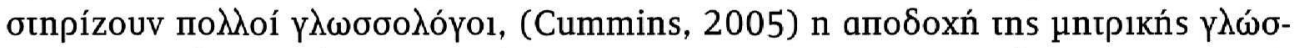

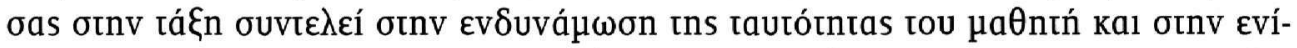

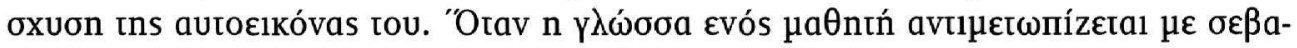

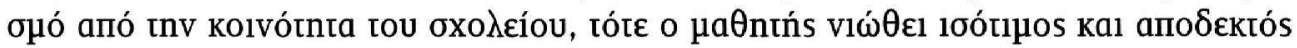

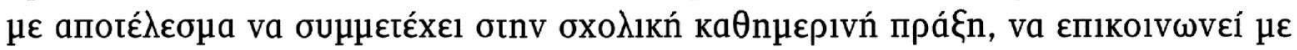

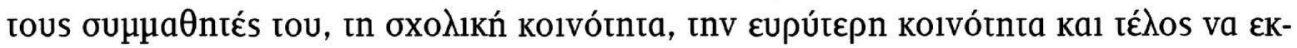

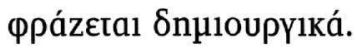

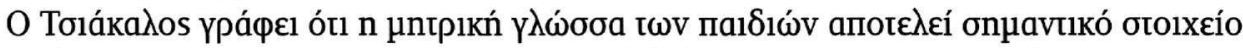

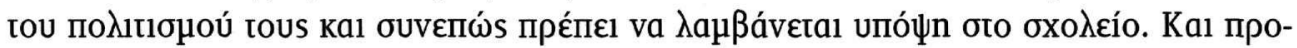

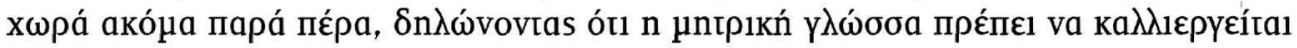

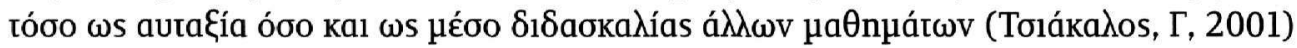

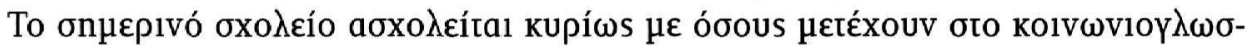

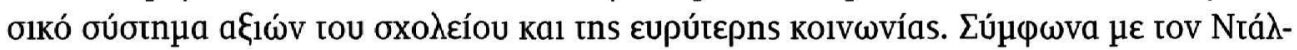

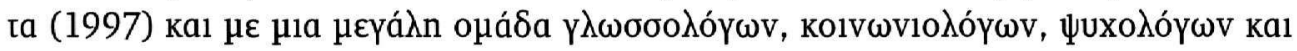

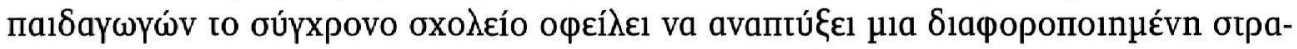

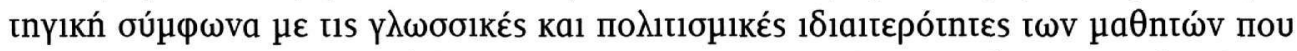

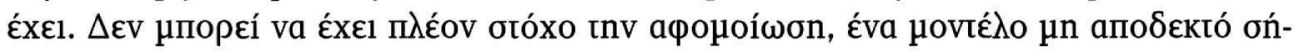

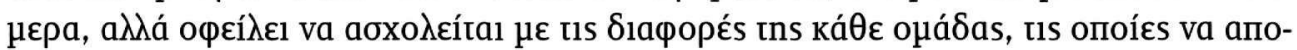

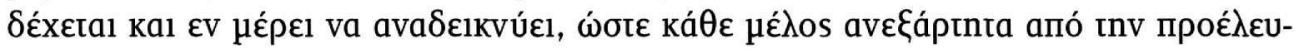

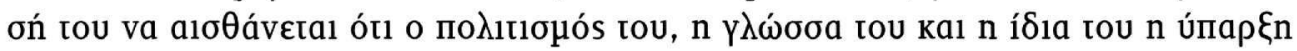

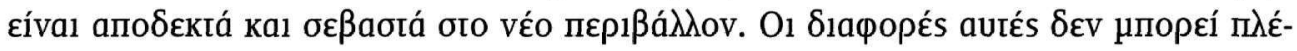

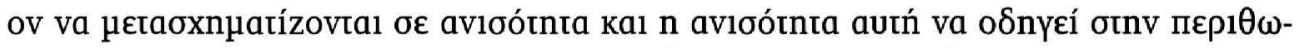

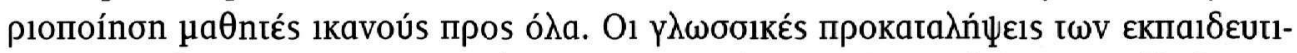

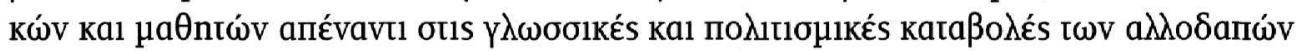

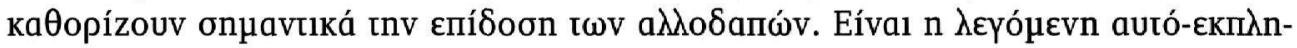

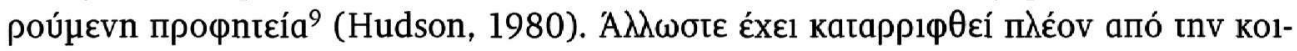

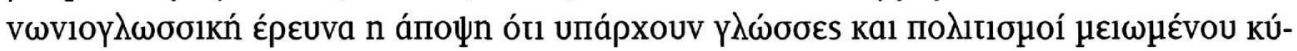

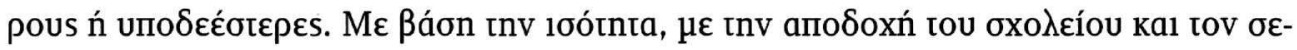

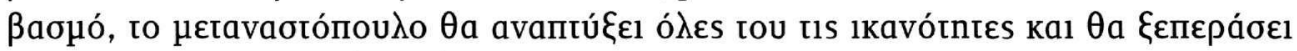

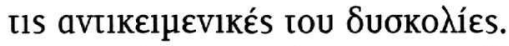

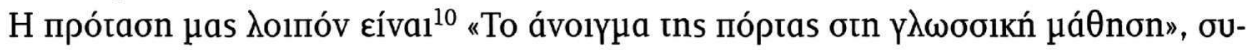

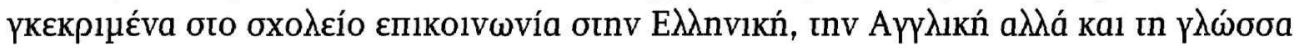

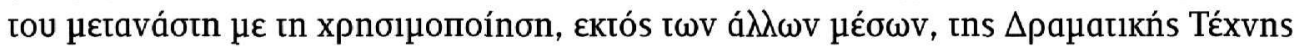

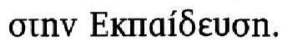

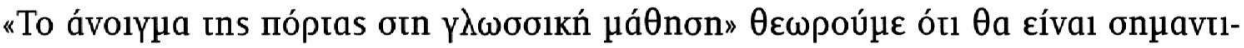

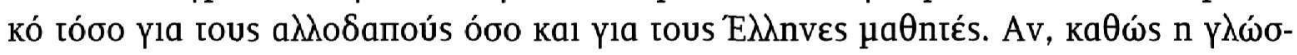

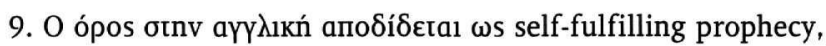

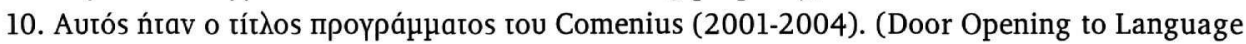
Learning). 


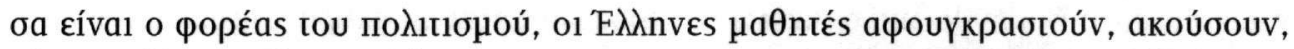

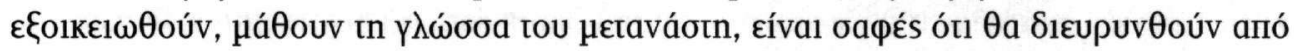

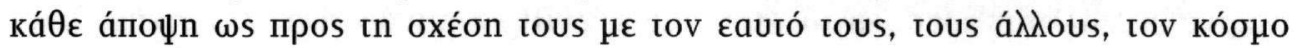
(Candelier, Macaire, 2001).

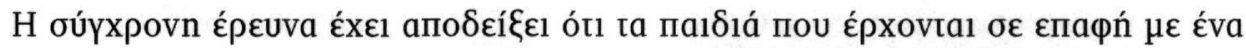

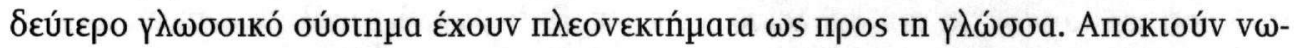

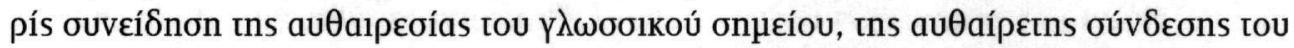

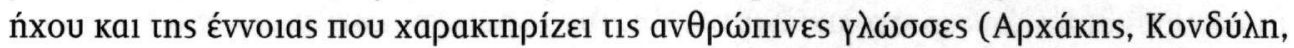

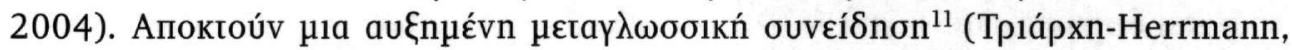

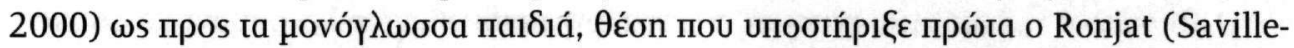
Troike, 1982), apүótepa o Leopold (McLauglin, 1978) kal o Vygotsky $(1962)^{12}$ ка1

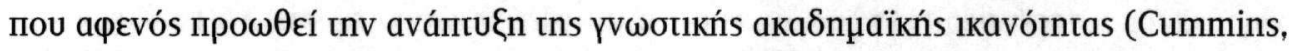

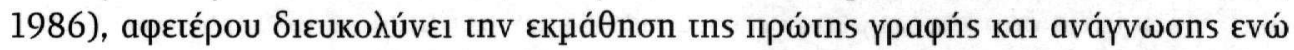

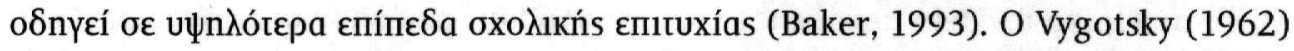

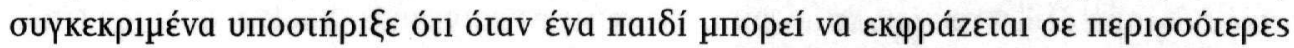

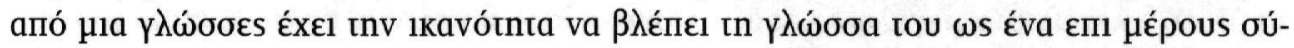

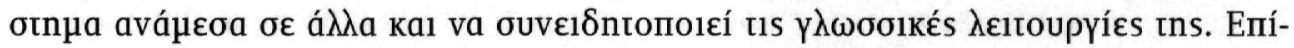

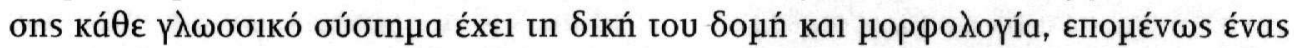

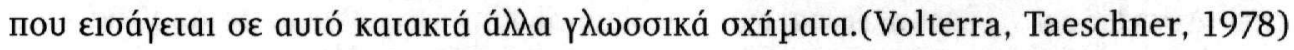

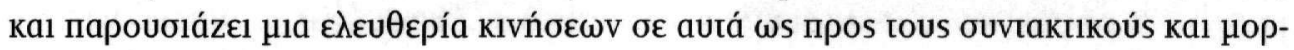
фоגoүıкоús kavóves.

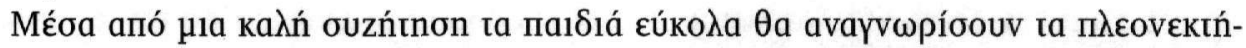

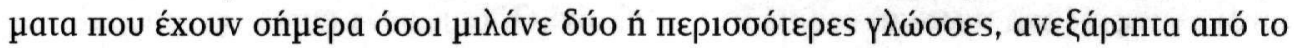
кúpos $\tau \omega \mathrm{v} \gamma \lambda \omega \sigma o \omega ́ v$ поU $\mu 1 \lambda a ́ v \varepsilon$.

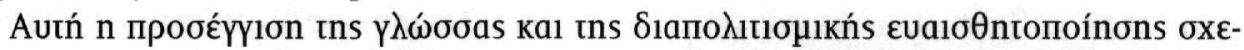

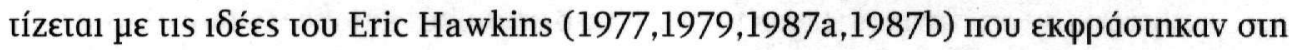

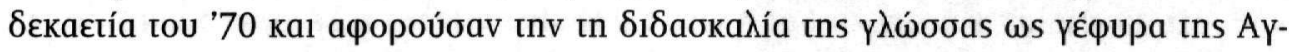

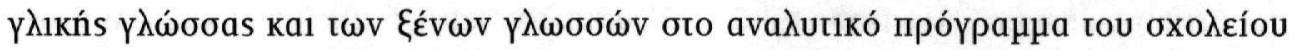

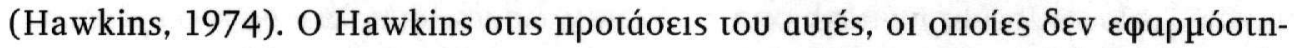

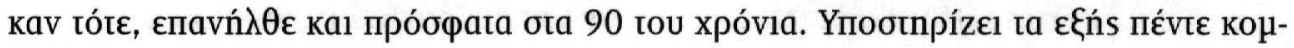

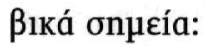

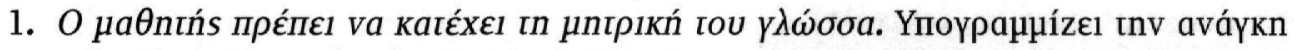

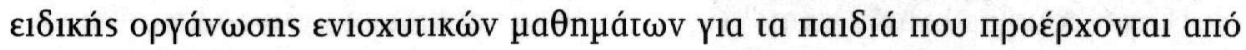

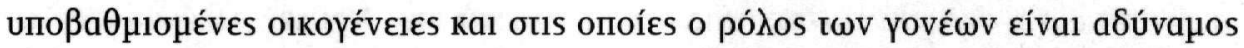

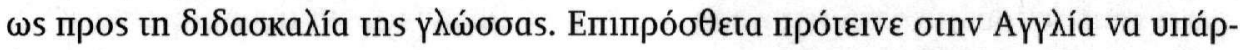

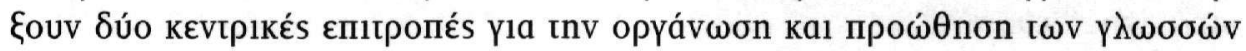

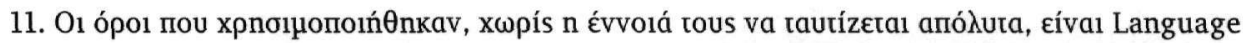

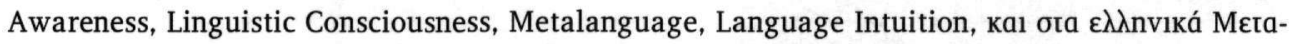

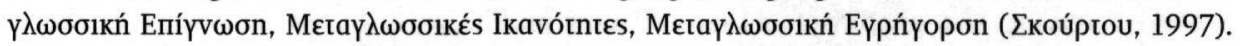

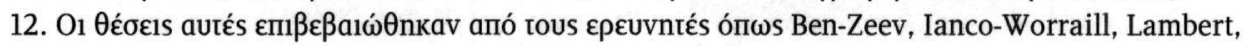
Tucket (1972), Cummins (1978b, 1986), Hakuta, et al (1987). 


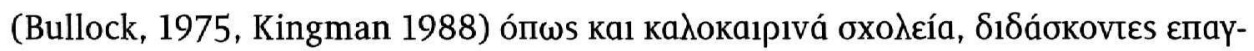

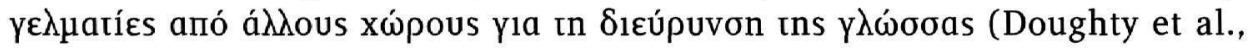

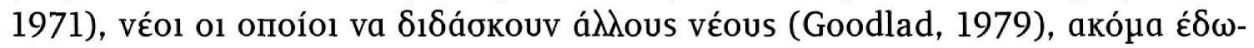

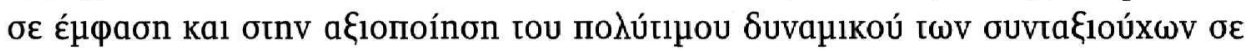
кá $\theta \varepsilon$ kolvómnta.

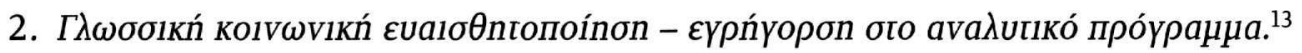

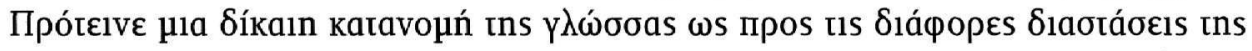

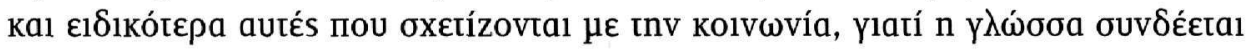

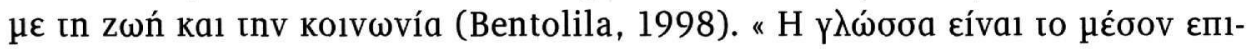

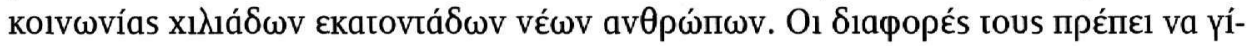

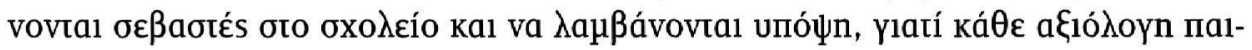

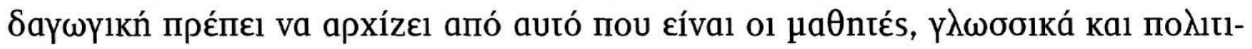
ou1кá» (Bentolila, 1998).

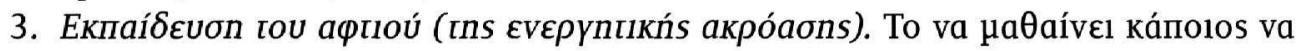

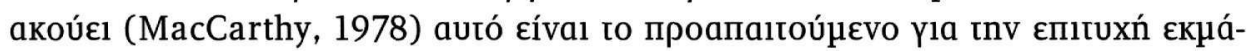

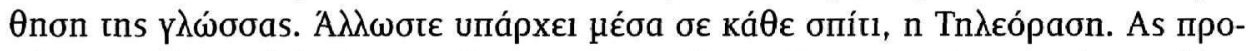

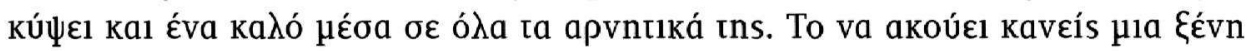

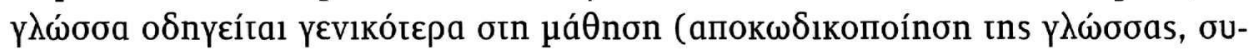

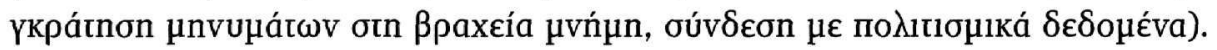

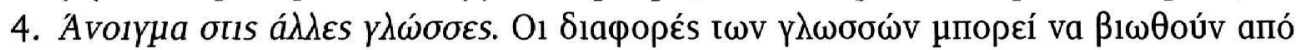

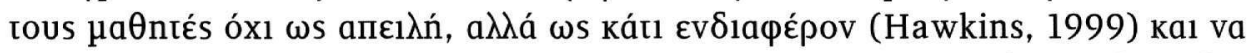

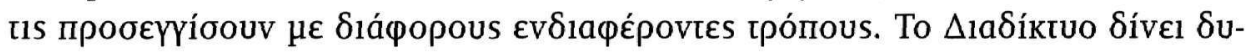

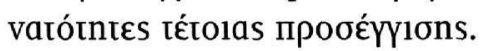

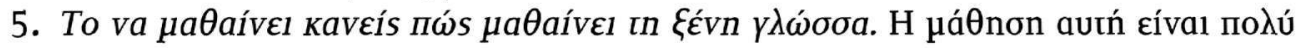

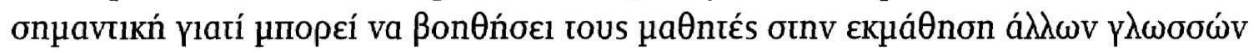

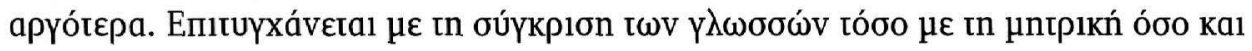

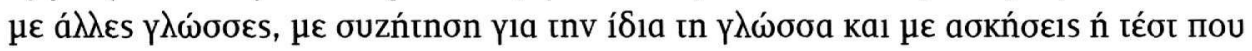

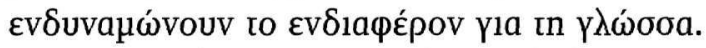

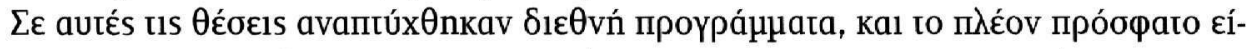

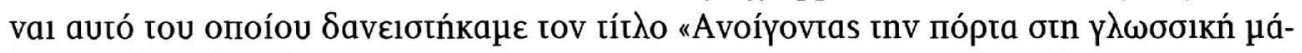
Өnon» (Opening the Door to Language Learning, www.opendoor2languages.net)

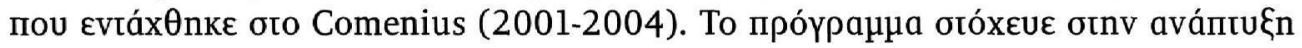

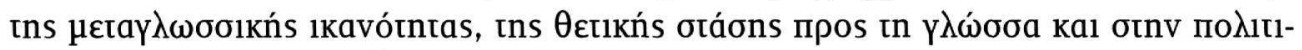

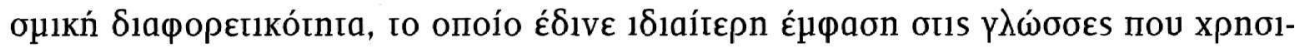

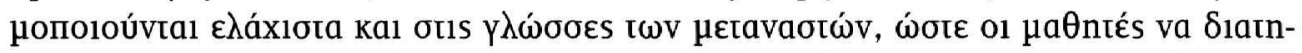

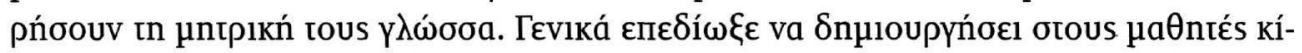

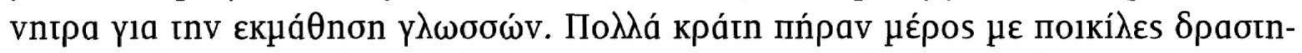

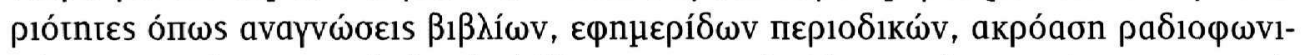

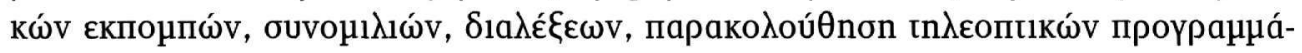

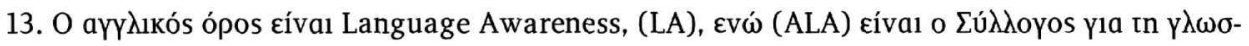

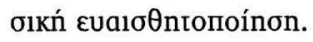




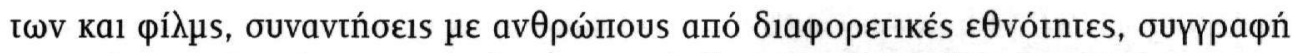

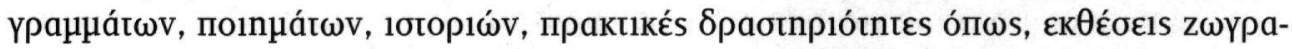

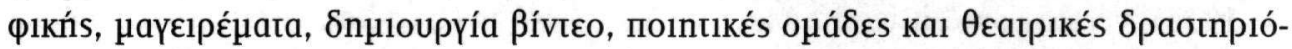

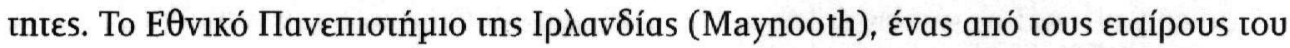

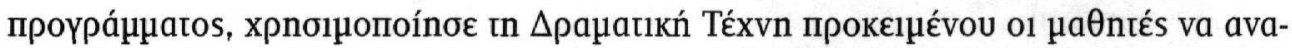

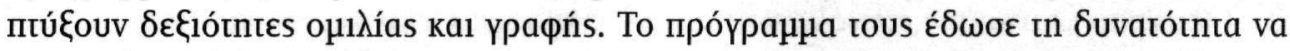

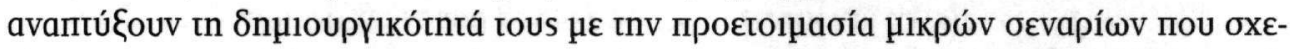

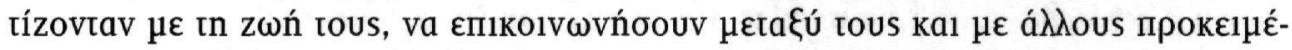

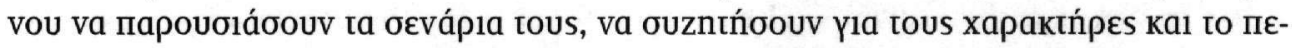

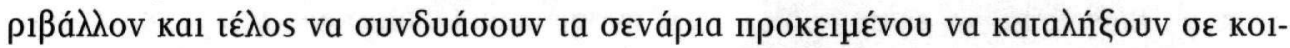

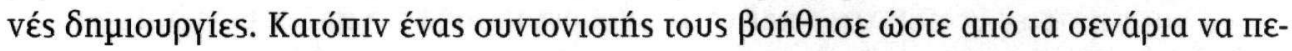

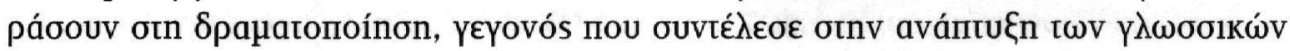

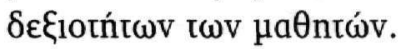

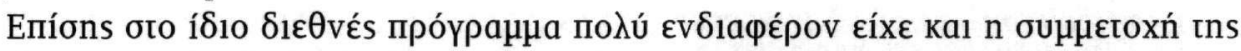

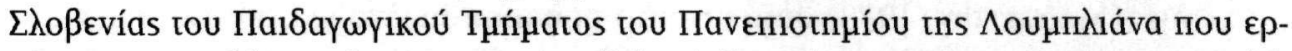

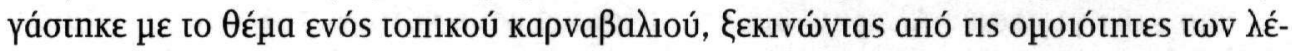

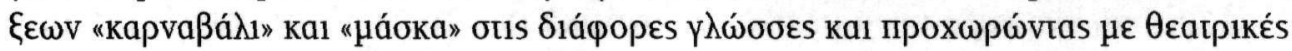
tદxvıkés (Fidler, Avsenik, Klavs, 2004).

( Пívakas - KARNEVAL - KARNEVAL - CARNAVAL - CARNEVALE - CARNIVAL MASKA - MASKE - MASQUE - MASCHERA - MASK)

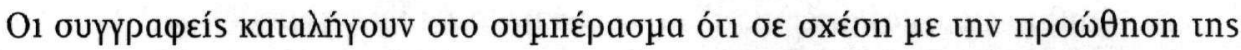

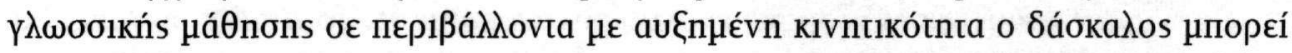

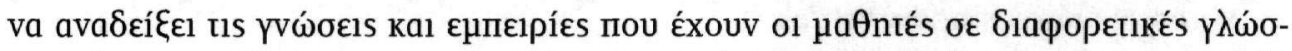

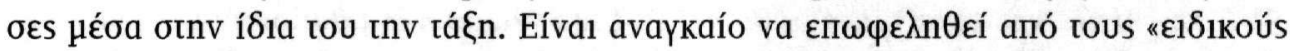

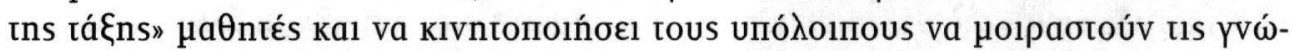

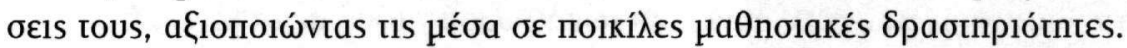

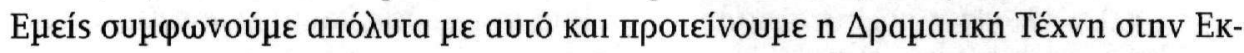

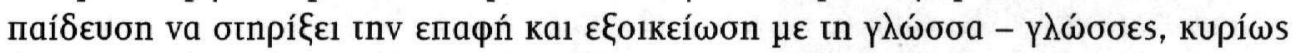

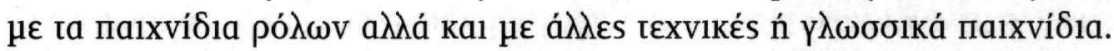

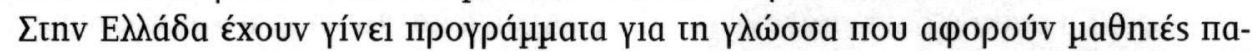

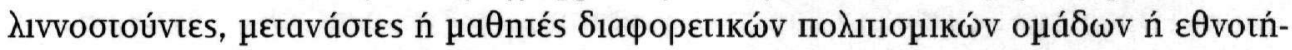

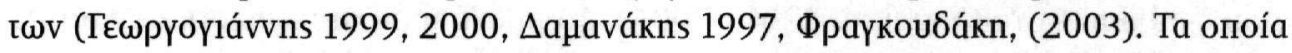

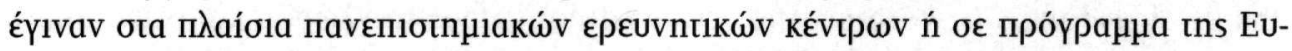

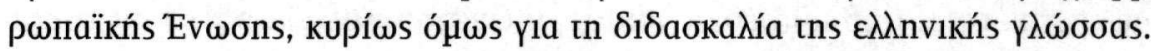

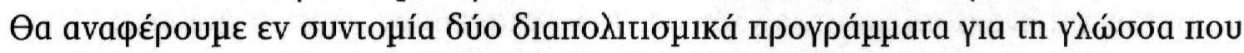

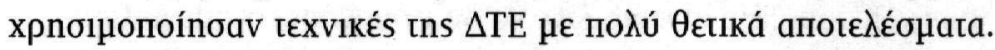

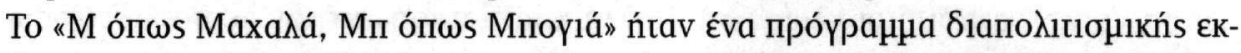

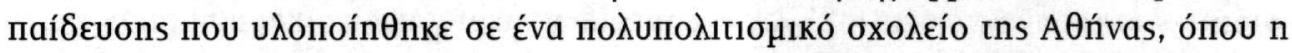

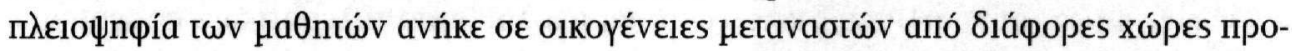

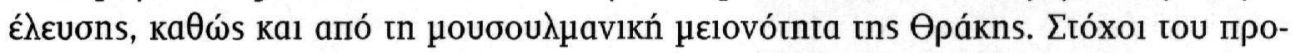

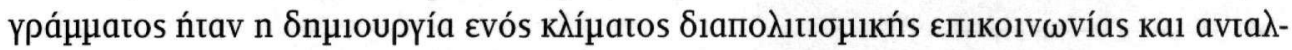

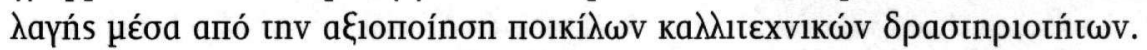

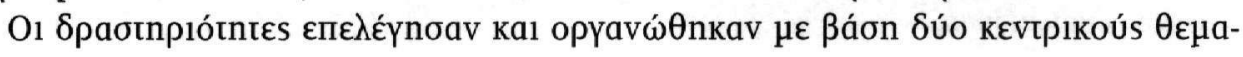




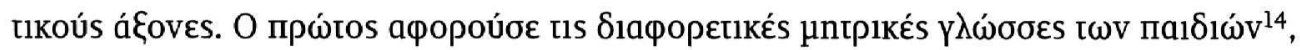

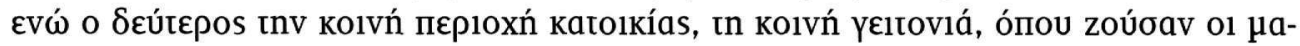
Ontés kal ol olkoүévelés tous (Máyos, 2004).

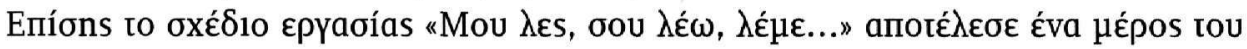

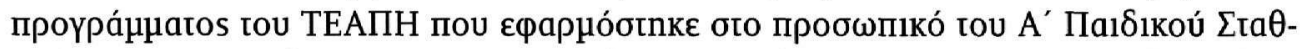

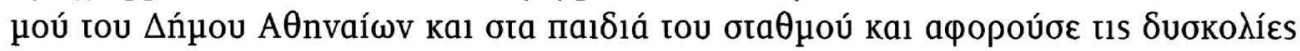

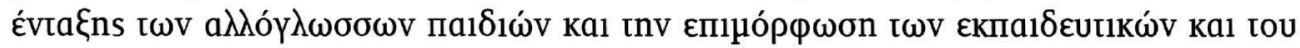

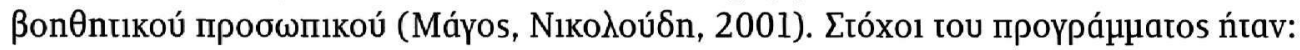

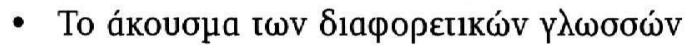

- H пробпá

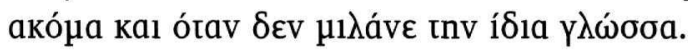

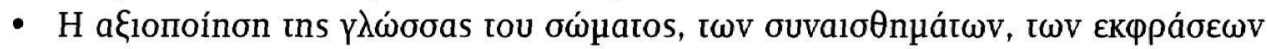

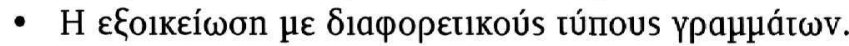

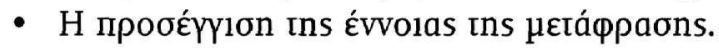

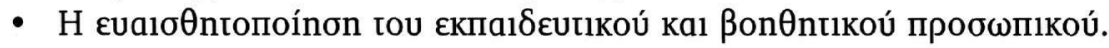

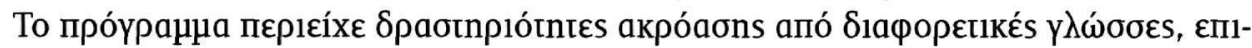

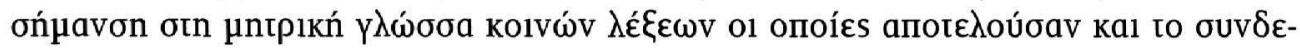

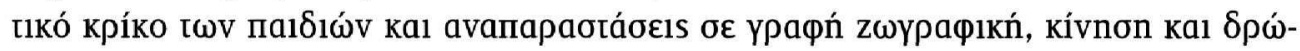

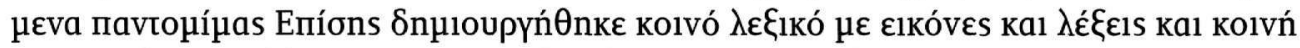

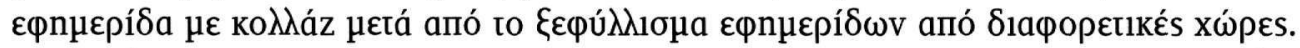

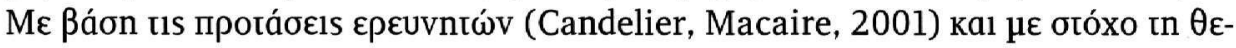

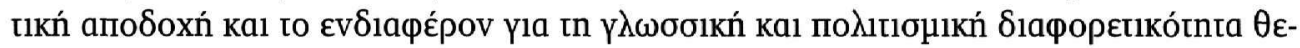

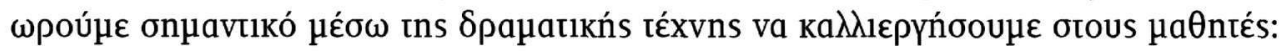

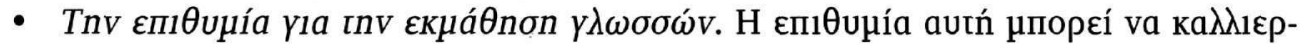

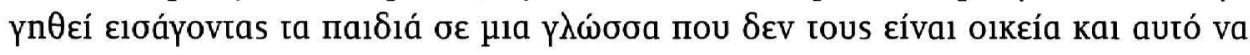

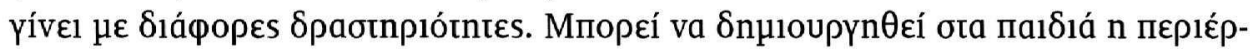

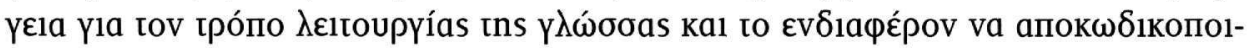

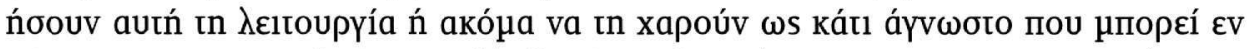

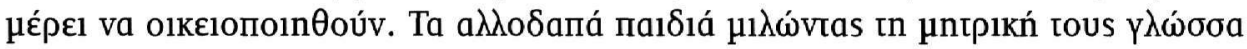

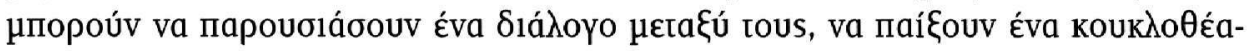

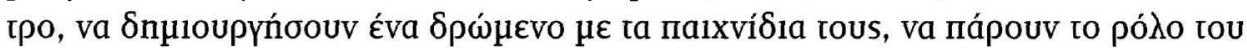

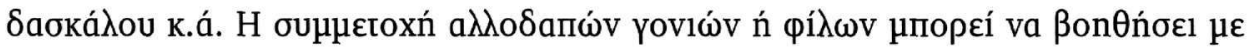

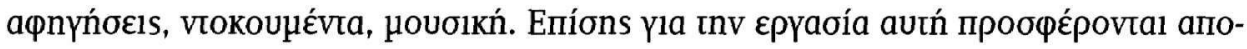

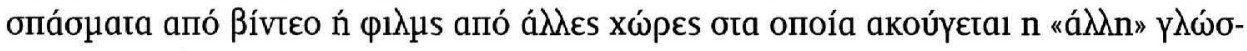

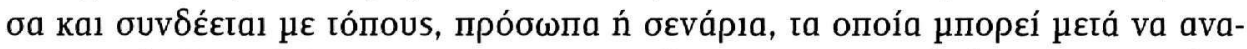

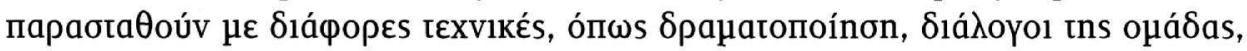

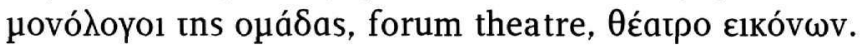

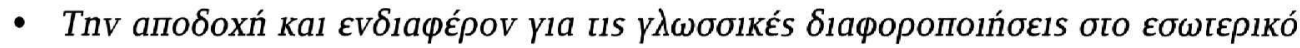

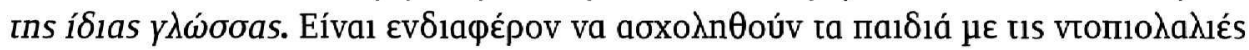

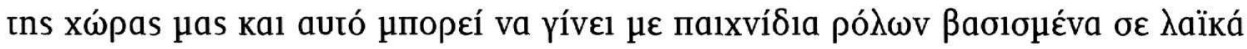

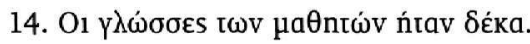




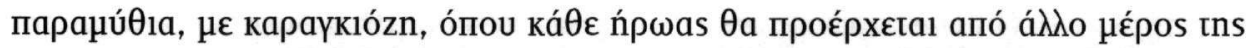

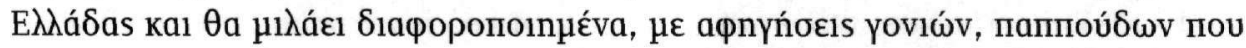

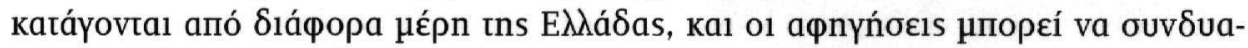

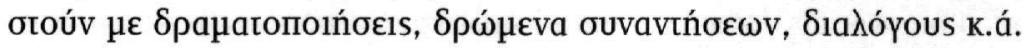

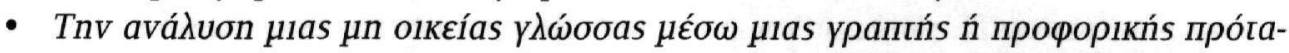

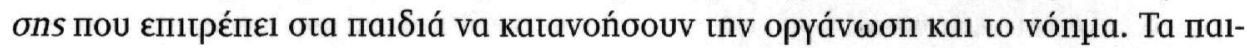

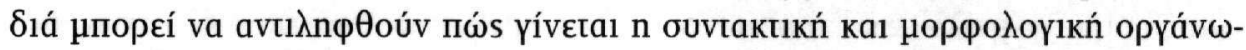

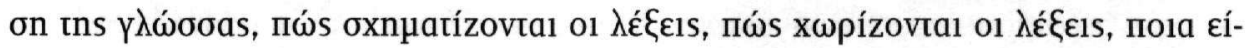

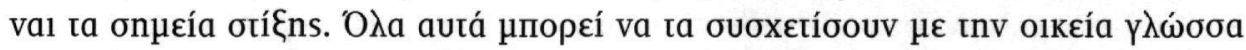

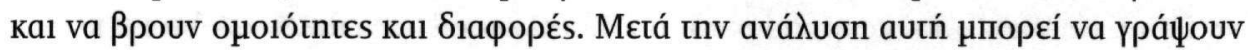

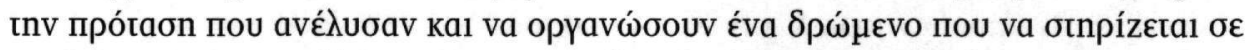

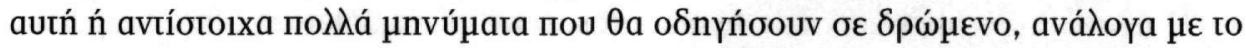

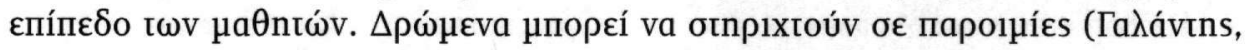

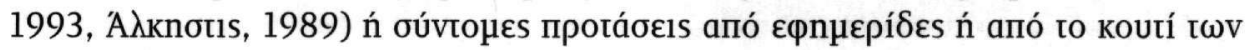

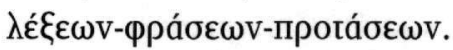

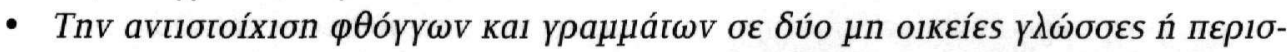

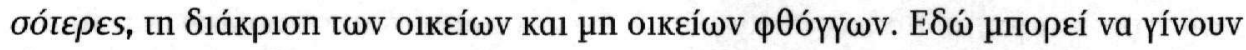

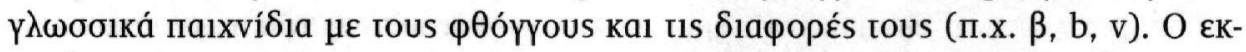

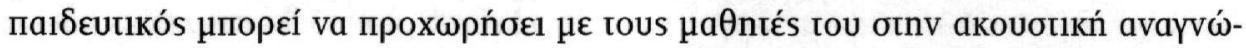

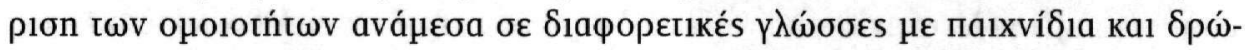

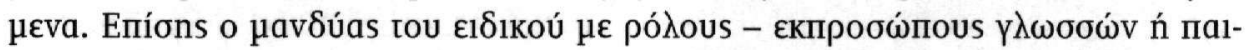

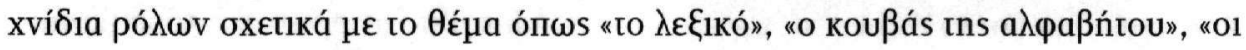

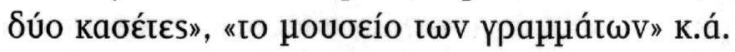

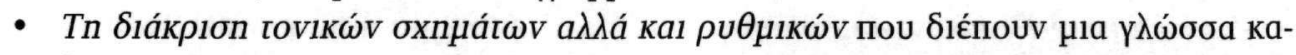

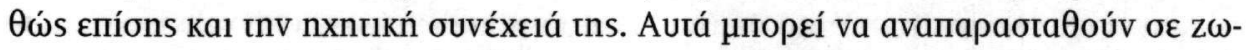

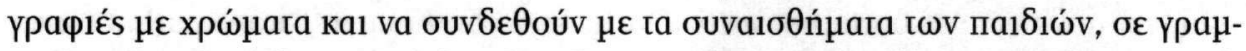

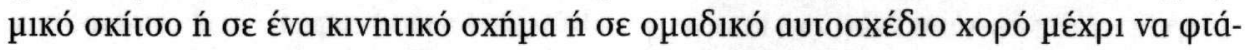

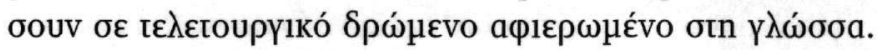

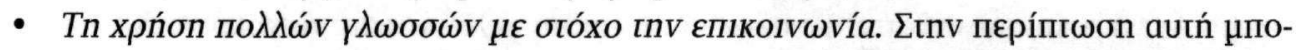

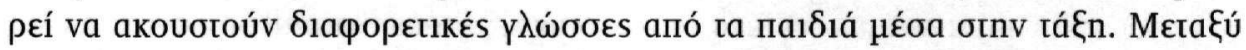

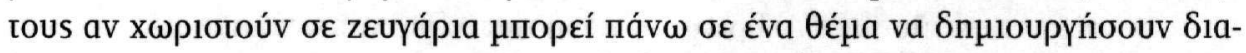

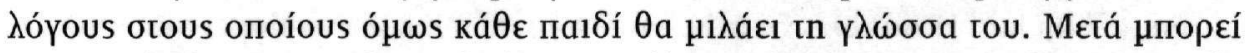

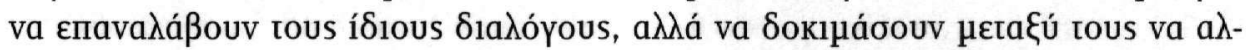

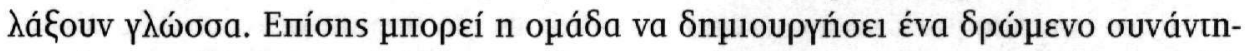

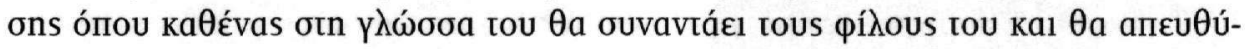

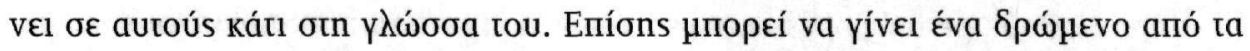

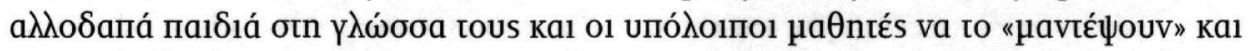

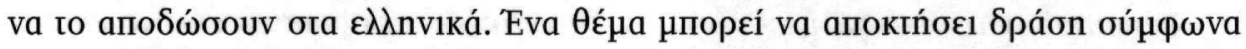

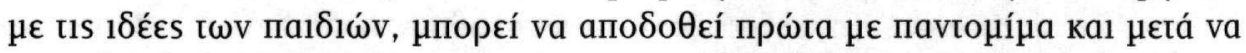

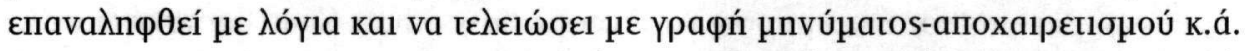

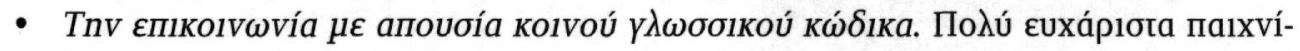

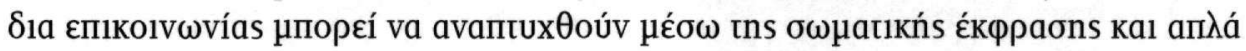

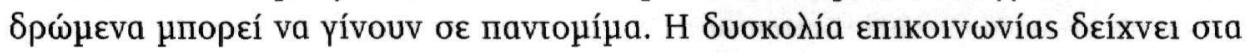




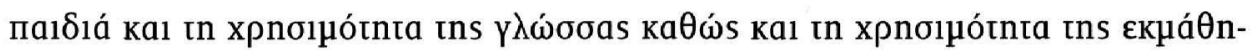

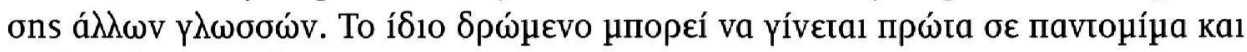

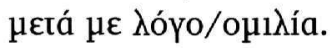

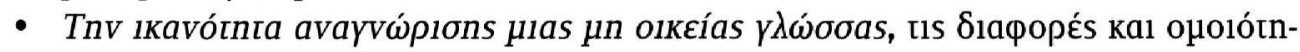

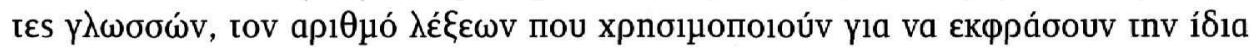

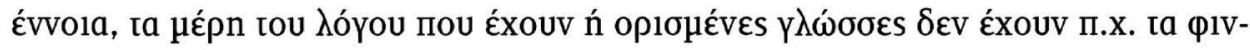

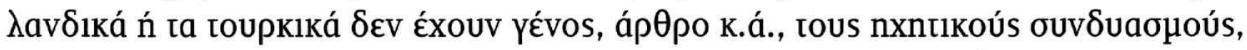

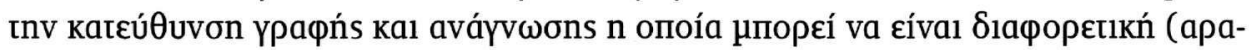

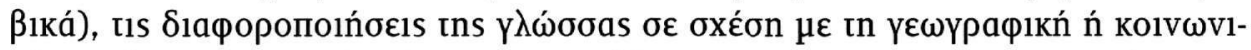

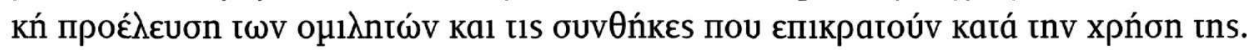

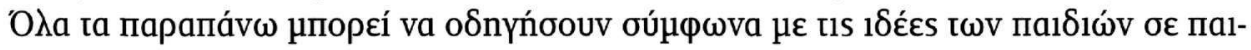

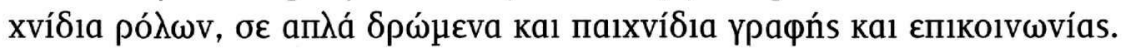

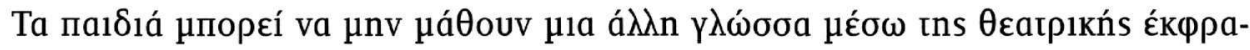

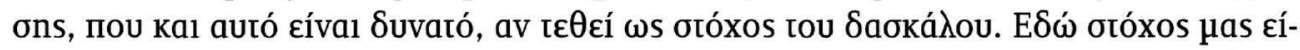

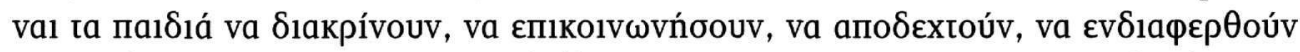

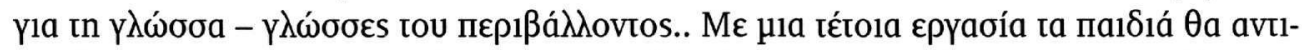

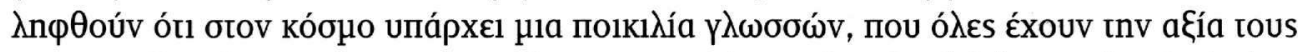

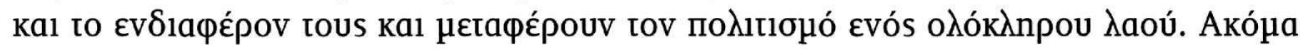

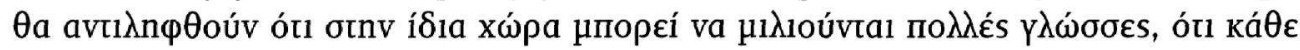

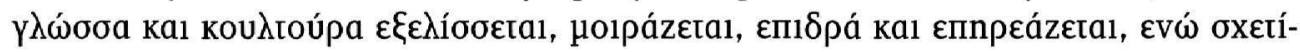

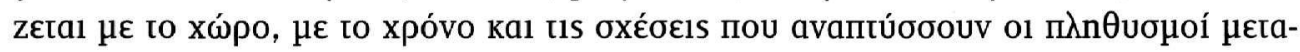

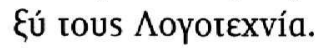

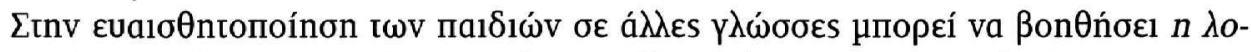

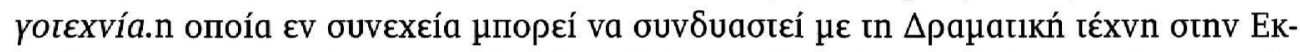

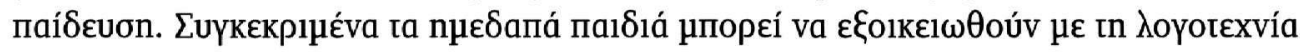

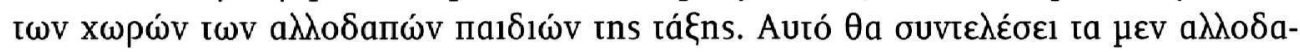

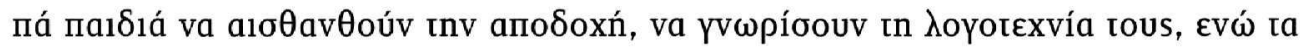

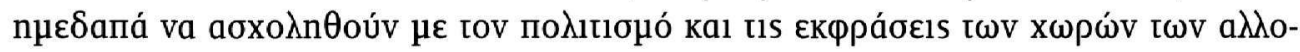

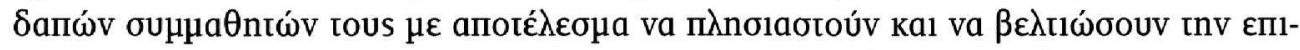

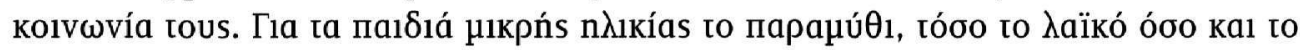

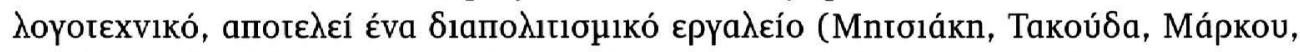

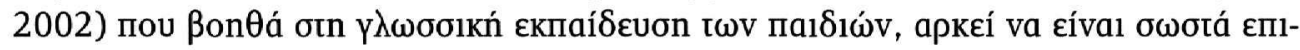

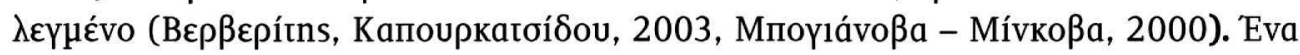

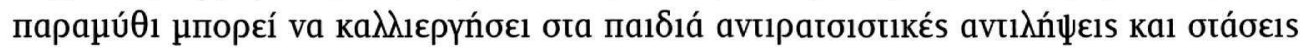

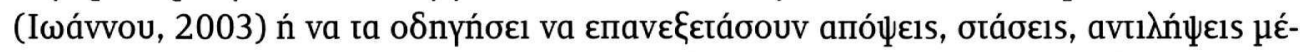

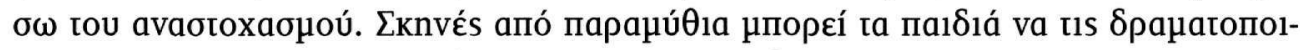

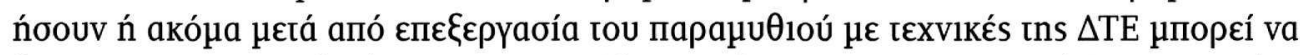

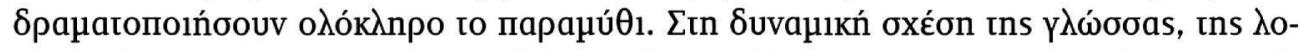

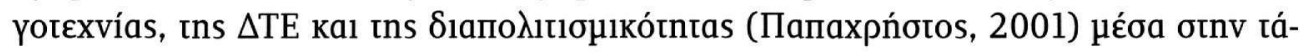

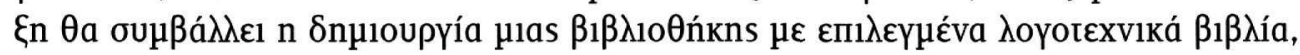

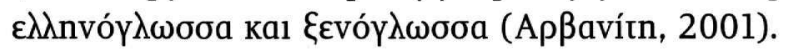

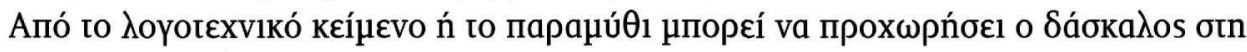

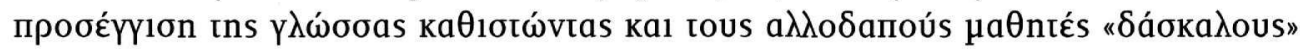




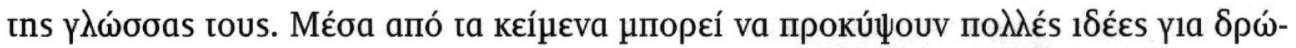

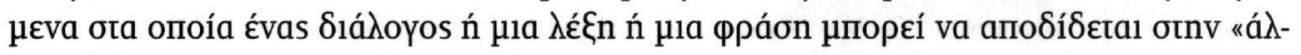

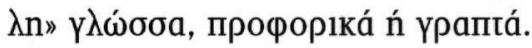

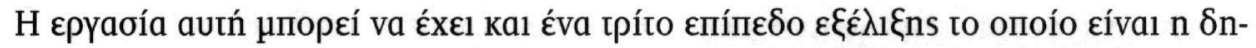

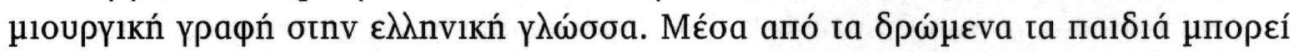

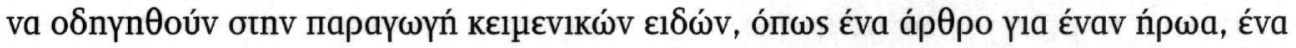

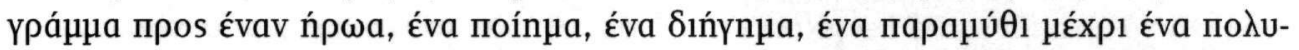

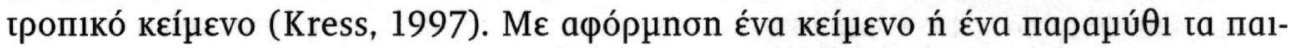

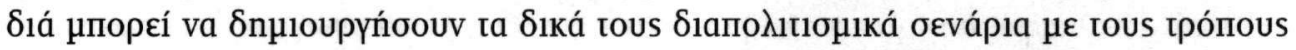

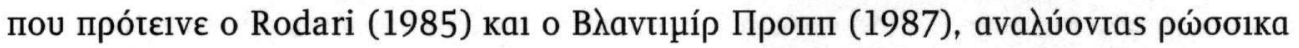

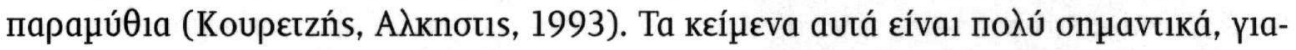

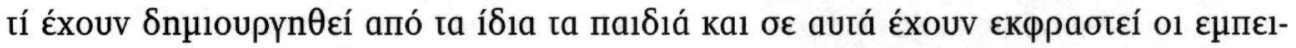

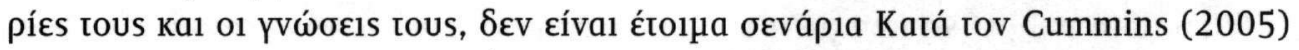

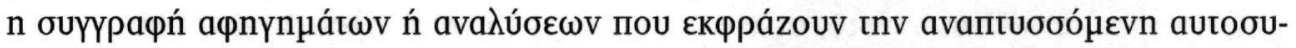

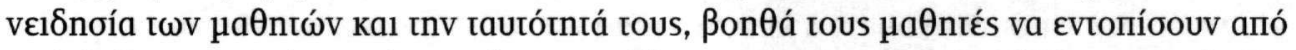

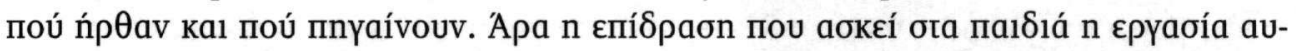

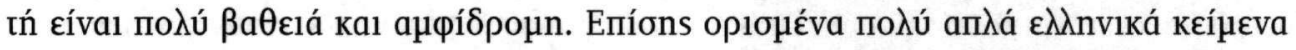

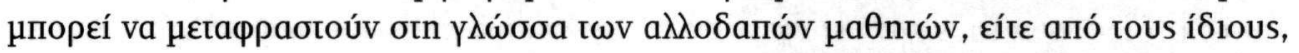

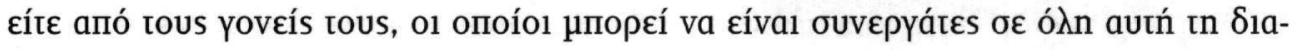

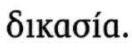

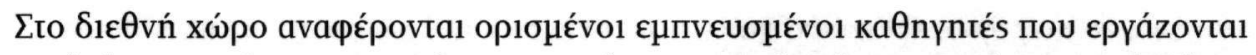

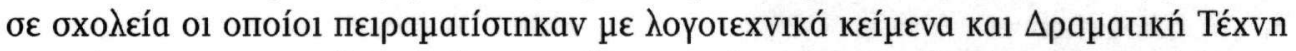

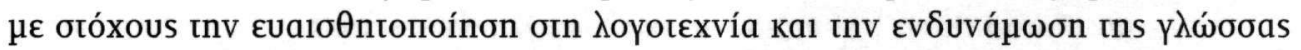

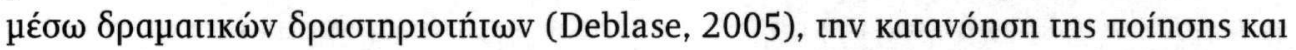

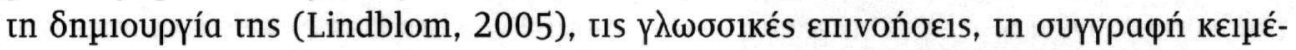

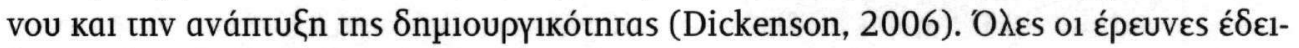

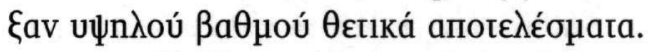

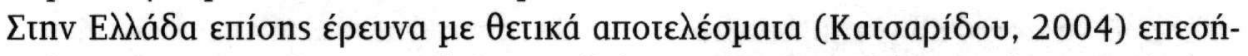

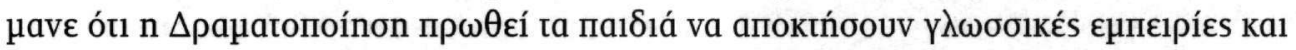

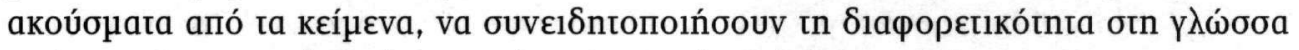

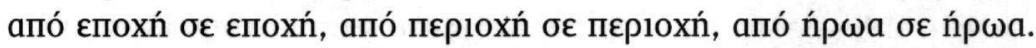

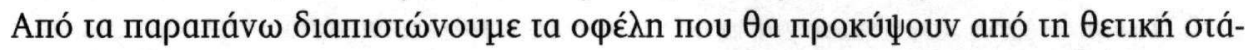

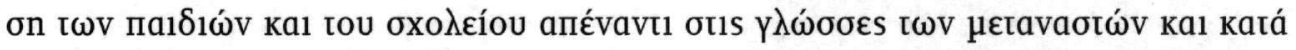

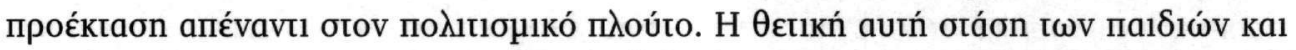

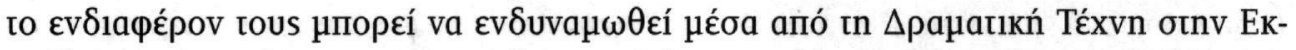

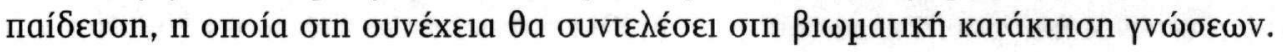

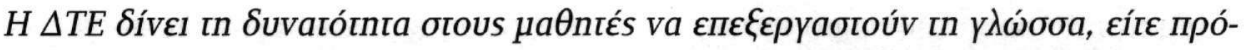

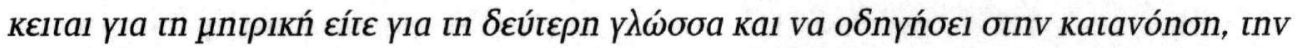

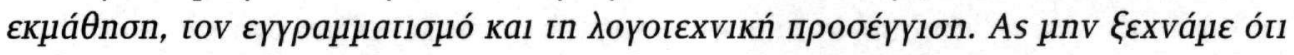

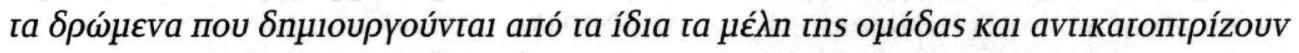

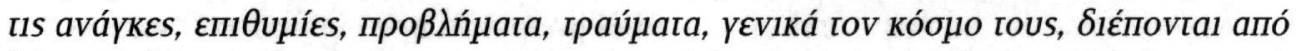

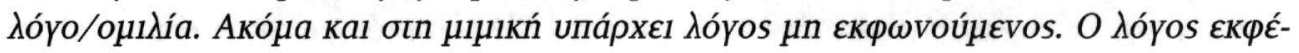




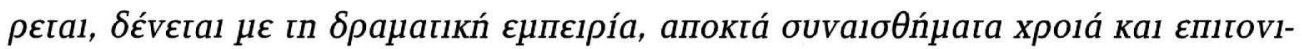

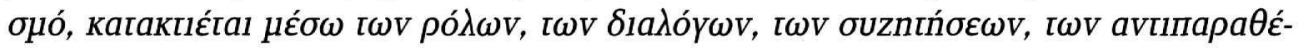

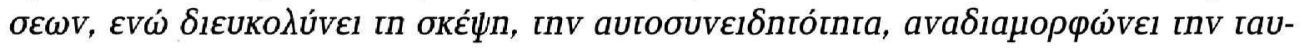

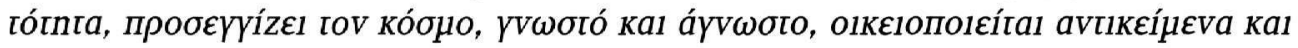

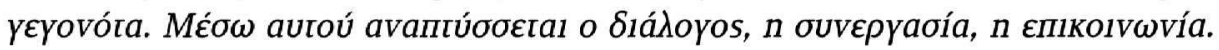

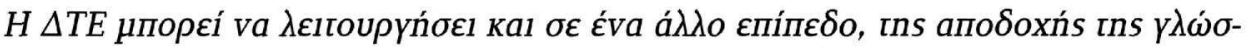

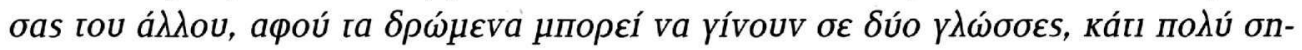

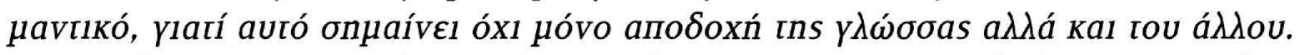

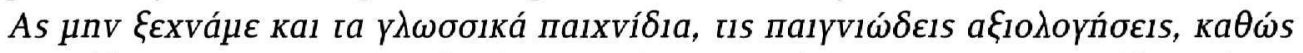

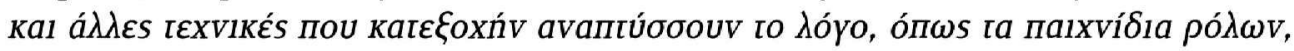

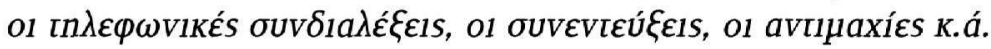

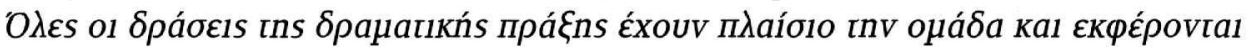

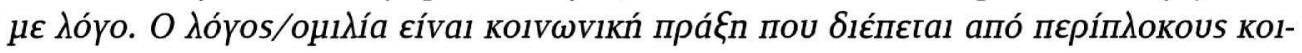

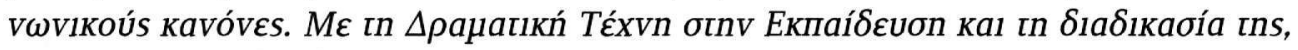

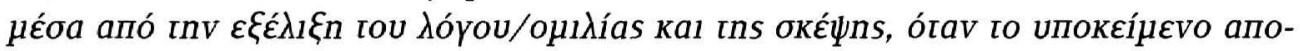

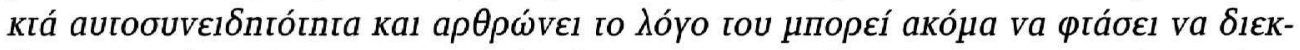

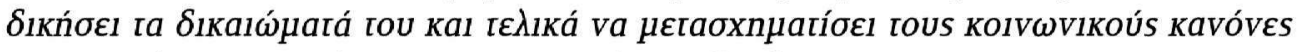

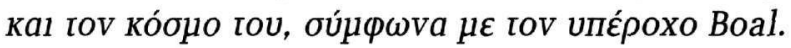

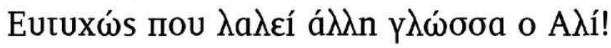

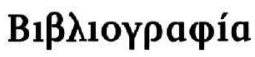

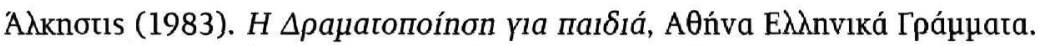

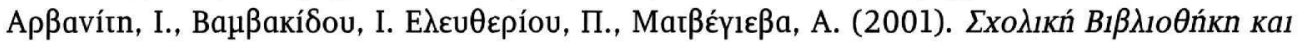

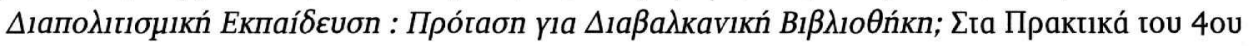

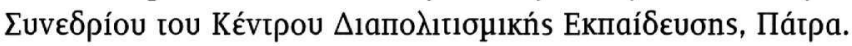

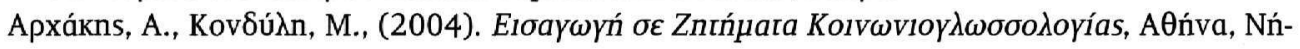
oos.

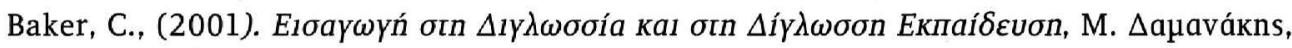

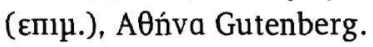

Bentolila, A., (1998). Les Faux Semblants du Franchais Branche'. The Learner and the School, Harmondsworth: Penguin.

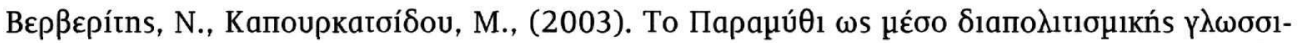

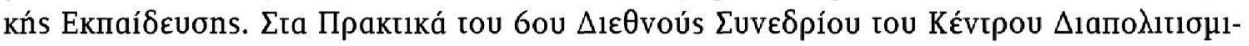
kńs

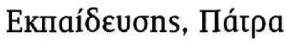

Bullock, A., (Chairman) (1975). A Language for Life. Report of Committee of Inquiry, London: HMSO

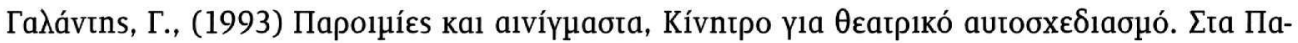

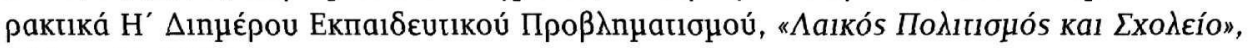
A日ńva, 22-23 Maïou.

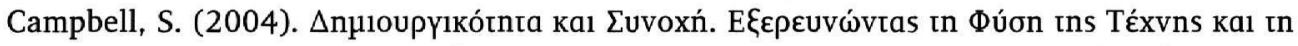

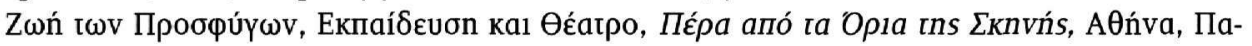

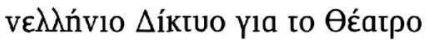


Candelier, M. \& Macaire, D. (2001). L'éveil aux langues à l'école primaire et la construction de compétences - pour mieux apprendre les langues et vivre dans une société multilingue et multiculturelle. oto L. Collés, J.-L. Dufays, G. Fabry \& C. Maeder (dir.), Actes de colloque de Louvain "Didactique des langues romanes: le développement des compétences chez l'apprenant", 495-506. Bruxelles, De Boeck - Duculot.

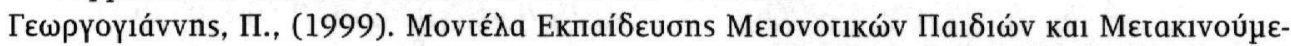

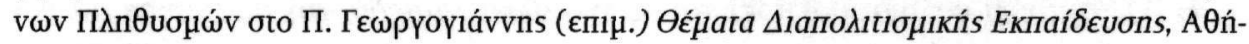
va, Gutenberg.

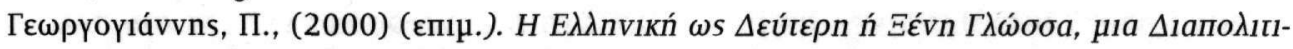

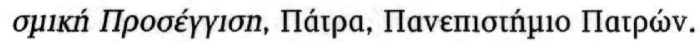

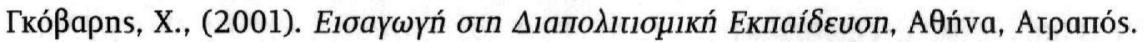

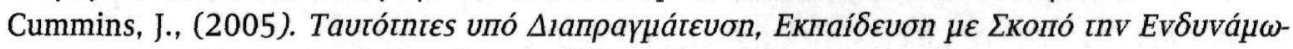

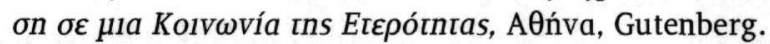

Cummins, J., (1978b). Bilingualism and the Development of Metalinguistic Awareness, Journal of Cross-Cultural Psychology, V., 9, pp. 131-149

Cummins, J., (1986). The Construct of Language Proficiency in Bilingual Education, oto J. Alatis, (ed.), Perspectives on Bilingualism and Bilingual Education,Washinghton, Georgetown University Press, pp. 209-231.

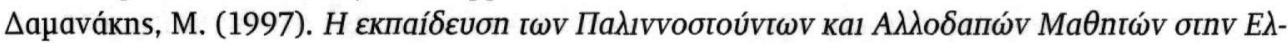

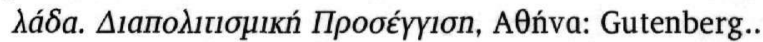

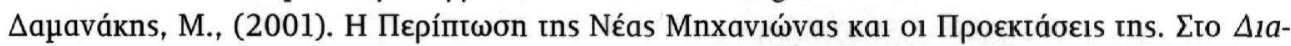

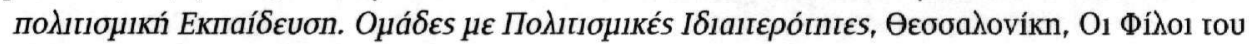

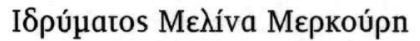

DeBlase, G., (2005). Teaching Literature and Language through Guided Discovery and Informal Classroom Drama, English Journal, V. 95, N. 1, pp. 29-32

Doughty, P., Pearce, J., Thornton, G., (1971). Language in Use, London: Edward Arnold

Fidler, S., Avsenik, B., Klavs, T. (2004). Language and International Awareness in Primary School, JaLing Comenius, 2001-2004.

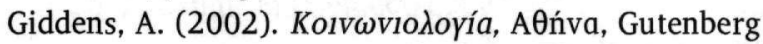

Goodlad, S., (1979). Learning by Teaching, Community Service Volunteers, London, NI 9NJ.

Hakuta, K., Ferdman, B., Diaz, R., (1987). Bilingualism and Cognitive Development: Three Perspectives, oto R. Sheldon (ed.), Advances in Applied Psycholinguistics, V. 1, Disorders of First Language Development, N. York, University Press, pp. 284-319.

Hawkins, E., (1974). Modern Languages in the Curriculum. Eto G. Perren (ed.), The Space Between: English and Foreign Languages at School, London: CILT (Centre for Information on Language Teaching and Research)

Hawkins, E. (1977). A Possible Way Forward. to P. Hoy (ed.), The Early Teaching of Modern Languages, London: Nuffield Foundation.

Hawkins, E. (1979). Language as a Curriculum Study. to G. Perren (ed.), The Mother Tongue and Other Languages in Education, NCLE Papers 2, London: CILT (Centre for Information on Language Teaching and Research).

Hawkins, E. (1987a). Modern Languages in the Curriculum, Cambridge: Cambridge University Press.

Hawkins, E. (1987b). Awareness of Language: An Intoduction, Cambridge, Cambridge University Press.

Hawkins, E. (1999). Foreign Language Study and Language Awareness, Language Awareness, V. 8 , No 3 \& 4 .

Hudson, R., (1980). Sociolinguistics, Cambridge, Cambridge University Press. 


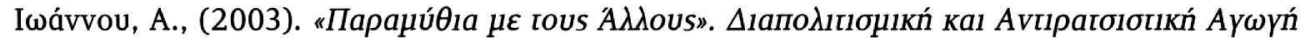

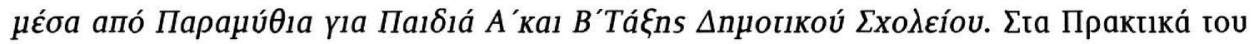

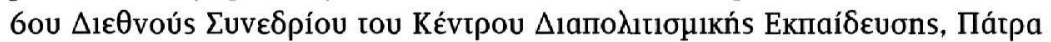

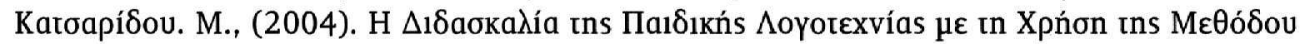

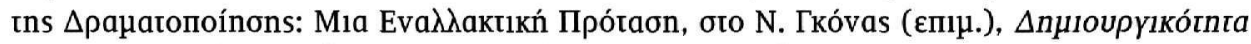

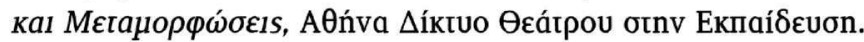

Kingman, J. (Chairman) (1988). Report of Commission of Inquiry into the Teaching of the English Language. London: HMSO.

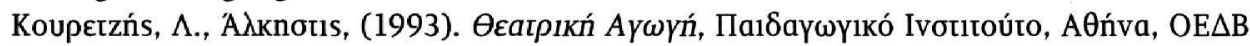

Kress, G. (1997). Before Writing. Rethinking the Paths to Literacy. London, Routledge

Lambert, W., Tucket, G., (1972), The Bilingual Education of Children, Newbury House, Rowley.

Lindblom, K., (2005). Teaching English in the World, English Journal, September, V., 95, N 1, p. 116

MacCarthy, P., (1978). The Teaching of Pronunciation. Cambridge: University Press.

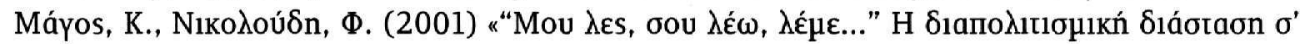

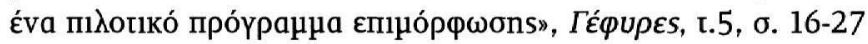

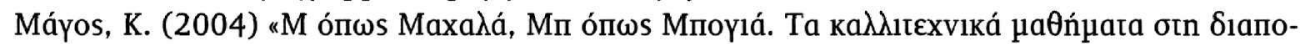

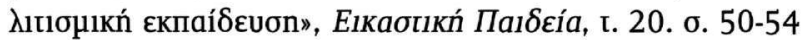

McLauglin, B., (1978). Second Language Acquisition in Childhood, N. Jersey, Hillsdale.

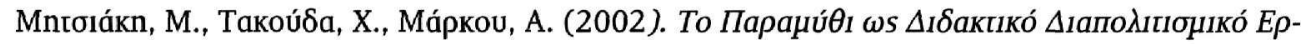

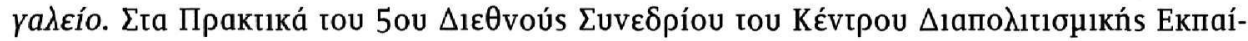

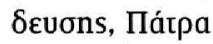

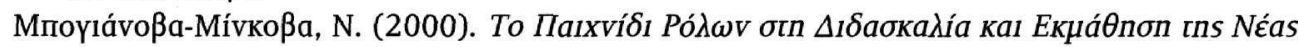

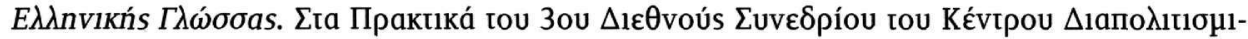

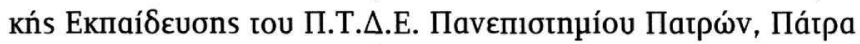

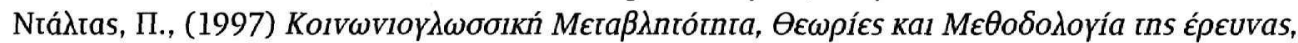
AӨńva, Eпıkaipótnta.

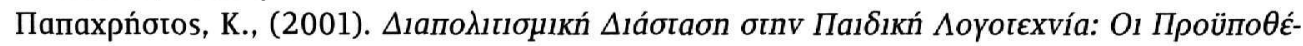

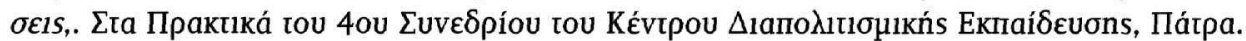

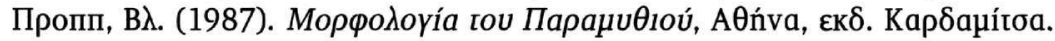

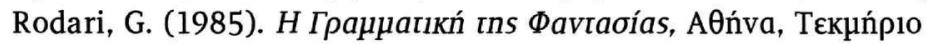

Saville-Troike, M., (1982). The Development of Bilingual and Bicultural Competence in Young Children, oto L. Katz (ed.), Current Topics in Early Childhood Education, Norwood, VD IV, pp. 1-16.

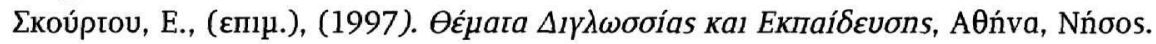

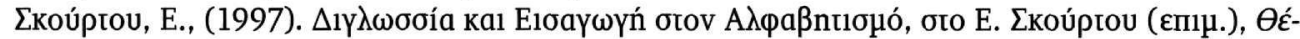

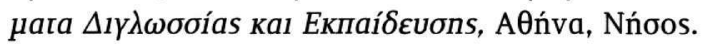

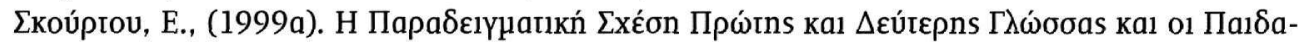

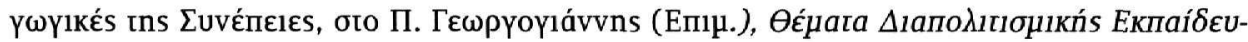
ons, AӨńva, Gutenberg.

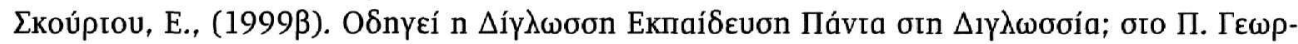

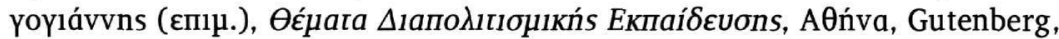

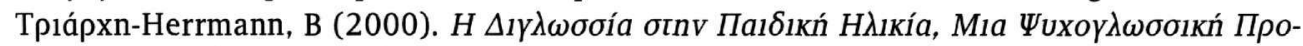

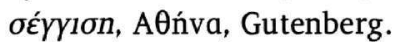

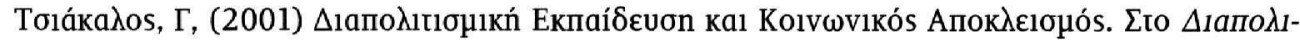

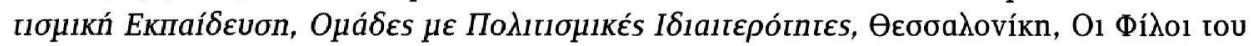

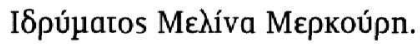


Turner, V., (1974). Dramas, Fields and Metaphors: Symbolic Action in Human Action Society, Ithaca, Cornwell University Press.

Volterra, V., Taeschner, T., (1978). The Acquisition and Development of Language by Bilingual Children, Journal of Child Language, V. 5, pp. 311-326

Vygotsky, L., (1962). Thought and Language, Cambridge, Massachusetts, MIT Press.

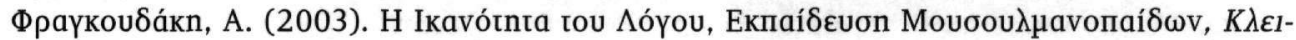

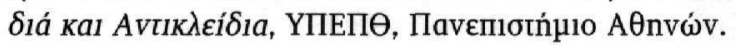

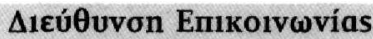

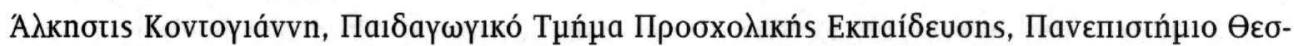

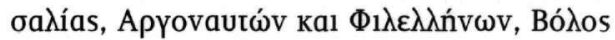

e-mail: alkistis@pre.uth.gr 